\title{
Stationary Pattern of a Ratio-Dependent Food Chain Model with Diffusion
}

\author{
Rui Peng \\ Junping Shi \\ Junping Shi \\ William \& Mary, jxshix@wm.edu \\ Mingxin Wang
}

Follow this and additional works at: https://scholarworks.wm.edu/aspubs

\section{Recommended Citation}

Peng, R., Shi, J., \& Wang, M. (2007). Stationary pattern of a ratio-dependent food chain model with diffusion. SIAM Journal on Applied Mathematics, 67(5), 1479-1503.

This Article is brought to you for free and open access by the Arts and Sciences at W\&M ScholarWorks. It has been accepted for inclusion in Arts \& Sciences Articles by an authorized administrator of W\&M ScholarWorks. For more information, please contact scholarworks@wm.edu. 


\title{
STATIONARY PATTERN OF A RATIO-DEPENDENT FOOD CHAIN MODEL WITH DIFFUSION*
}

\author{
RUI PENG ${ }^{\dagger}$, JUNPING SHI ${ }^{\ddagger}$, AND MINGXIN WANG ${ }^{\S}$
}

\begin{abstract}
In the paper, we investigate a three-species food chain model with diffusion and ratio-dependent predation functional response. We mainly focus on the coexistence of the three species. For this coupled reaction-diffusion system, we study the persistent property of the solution, the stability of the constant positive steady state solution, and the existence and nonexistence of nonconstant positive steady state solutions. Both the general stationary pattern and Turing pattern are observed as a result of diffusion. Our results also exhibit some interesting effects of diffusion and functional responses on pattern formation.
\end{abstract}

Key words. food chain model, diffusion, ratio-dependent functional response, stationary pattern, Turing pattern, steady state solution

AMS subject classifications. 35J55, 92C15, 92D40

DOI. $10.1137 / 05064624 \mathrm{X}$

1. Introduction. Understanding of spatial and temporal behaviors of interacting species in ecological systems is a central issue in population ecology. One aspect of great interest for a model with multispecies interactions is whether the involved species can persist or even stabilize at a coexistence steady state. In the case where the species are homogeneously distributed, this would be indicated by a constant positive solution of an ordinary differential equation (ODE) system. In the spatially inhomogeneous case, the existence of a nonconstant time-independent positive solution, also called stationary pattern, is an indication of the richness of the corresponding partial differential equation (PDE) dynamics. In recent years, stationary pattern induced by diffusion has been studied extensively, and many important phenomena have been observed.

In particular, starting with Turing's seminal 1952 paper [34], diffusion has been regarded as the driving force of the spontaneous emergence of spatiotemporal structure in a variety of nonequilibrium situations. To verify the influence of diffusion on this aspect, in the past decades, biologists and applied mathematicians have proposed a number of models, and much work has been devoted to the investigation of the existence of stationary pattern in chemical and biological dynamics theoretically as well as numerically. For example, chemical models include the activator-inhibitor Gierer-Meinhardt model [10, 23], the Sel'kov model [7, 35], the Gray-Scott model [32, 37 , the Brusselator model [3, 30], the Noyes-Field model for Belousov-Zhabotinskii

${ }^{*}$ Received by the editors November 29, 2005; accepted for publication (in revised form) April 26, 2007; published electronically August 24, 2007.

http://www.siam.org/journals/siap/67-5/64624.html

$\dagger$ Corresponding author. Institute of Nonlinear Complex System, College of Science, China Three Gorges University, Yichang, 443002, Hubei, People's Republic of China (pengrui_seu@163.com). The work of this author was supported by the Scientific Research Projects of Hubei Provincial Department of Education Q200713001.

${ }^{\ddagger}$ Department of Mathematics, College of William and Mary, Williamsburg, VA 23187-8795 and Department of Mathematics, Harbin Normal University, Harbin, 150080, Helongjiang, People's Republic of China (jxshix@wm.edu). The work of this author was partially supported by US-NSF grants DMS-0314736 and EF-0436318, NSFC grant 10671049, and a Longjiang scholar grant.

$\S$ Department of Mathematics, Southeast University, Nanjing, 210018, Jiangsu, People's Republic of China (mxwang@seu.edu.cn). The work of this author was supported by NSFC grant 10471022. 
reaction [29], and the chemotactic diffusion model [4, 18, 19, 22, 24, 37], and biological models include the competition model $[14,20]$ and the predator-prey model $[8,15$, $16,27,28,31,36]$ (see also the references therein).

In his original paper [34], Turing proposed the notion of diffusion-driven instability (also called Turing instability) in his attempt at modeling, among other things, the regeneration phenomenon of hydra - one of the earliest examples of morphogenesis. That is, Turing claimed that the formation of spatial pattern during morphogenesis could be explained in terms of the instability of a homogeneous steady state solution to a reaction-diffusion network describing the growth and movement of a set of morphogens. Turing's original work was primarily concerned with the stability analysis of the uniform steady state solution of the system for the interacting morphogens.

In biology and chemistry, the more interesting question, however, is whether the spatially inhomogeneous solution may be generated by such instability. Strikingly, in some cases, Turing instability can indeed lead to stationary pattern (also called Turing pattern), a fascinating phenomenon in nonlinear science, which has been found in various mechanisms $[4,18,26,27,28,30,35,36,37]$. While linear stability analysis of the homogeneous steady state is a straightforward method for calculating conditions for the onset of Turing instability, the analysis of the existence of resulting nonhomogeneous steady states is mathematically challenging. In this paper, it is the question of the existence of nonhomogeneous steady states that we focus on.

In the present work, we will investigate a coupled reaction-diffusion food chain model with ratio-dependent functional response and analyze the coexistence of the three species. We attempt to further understand the influences of diffusion and functional responses on pattern formation. As a consequence, the existence and nonexistence results for nonconstant positive steady state solutions to this system indicate that stationary pattern arises as the diffusion coefficients enter into certain regions. In other words, diffusion does help to create stationary pattern. For this model, we also show that Turing instability occurs and prove the generation of Turing pattern in some cases.

On the other hand, our results also demonstrate that diffusion and functional response can become determining factors in the formation of pattern. Although our model is very different from the one considered by Lou, Martinez, and Ni in [20], their interesting observation that the introduction of a new species may qualitatively change the pattern structure of the original system is again present in our study. At the same time, our work corroborates recent numerical results implemented by Alonso, Bartumeus, and Catalan in [2]. We refer the reader to more detailed discussions in section 6 .

Our paper is organized as follows. In section 2, we propose our mathematical model. In section 3, we discuss the persistence and stability of the unique constant positive steady state for the ODE and PDE systems. In section 4, we consider the nonexistence of nonconstant positive steady state solutions, while section 5 is devoted to the existence of nonconstant positive steady state solutions. In section 6 , from the biological viewpoint we make some comments on our studies, indicating some interesting influences of diffusion and functional responses on pattern formation. Finally, in the appendix, we analyze some conditions, which are imposed in section 5 to obtain the nonconstant positive steady state solutions to the PDE system.

2. The derivation of the mathematical model. Numerous examples from biological control indicate that the classical prey-dependent predator-prey model is often contrary to actual observations, such as the well-known paradox of enrichment formulated by Rosenzweig [33]. The theory of Rosenzweig states that enriching a 
predator-prey system (increasing the prey's carrying capacity) will cause an increase in the equilibrium density of the predator but not in that of the prey; it will destabilize the positive equilibrium as the prey's carrying capacity increases, and thus will increase the possibility of stochastic extinction of the predator. Recently there is growing evidence that in some situations, especially when predators have to search, share, and compete for food, a more suitable general predator-prey model should be a so-called ratio-dependent one (namely, the functional responses are ratio-dependent). Roughly speaking, this model states that the per capita predator growth rate should be a function of the ratio of prey to predator abundance (see, e.g., [1]).

In the case of multiple species interaction, the prey-dependent models such as those studied in $[5,9,11,17]$, while mathematically interesting, inherit the mechanism that generates the factitious paradox of enrichment and fail to produce the often observed extinction dynamics resulting in the collapse of the system. Consequently, a ratio-dependent food chain model, which is an ODE system with three equations whose species are hence assumed to be spatially homogeneous, was proposed by Hsu, Hwang, and Kuang in [13] to describe the growth of plant, pest, and top predator.

More precisely, the authors of [13] considered the following three-trophic-level food chain system with ratio-dependent functional response:

$$
\left\{\begin{array}{rlrl}
\frac{\mathrm{d} u_{1}}{\mathrm{~d} t} & =r u_{1}\left(1-\frac{u_{1}}{k}\right)-\frac{1}{\eta_{1}} \frac{m_{1} u_{1} u_{2}}{u_{1}+c_{1} u_{2}}, & & t>0 \\
\frac{\mathrm{d} u_{2}}{\mathrm{~d} t}=\frac{m_{1} u_{1} u_{2}}{u_{1}+c_{1} u_{2}}-b_{1} u_{2}-\frac{1}{\eta_{2}} \frac{m_{2} u_{2} u_{3}}{u_{2}+c_{2} u_{3}}, & & t>0 \\
\frac{\mathrm{d} u_{3}}{\mathrm{~d} t}=\frac{m_{2} u_{2} u_{3}}{u_{2}+c_{2} u_{3}}-b_{2} u_{3}, & & t>0, \\
u_{1}(0)>0, \quad u_{2}(0)>0, \quad u_{3}(0)>0, &
\end{array}\right.
$$

where $u_{i}(i=1,2,3)$ are the respective population densities of prey, predator, and top predator. For $i=1,2, \eta_{i}, m_{i}, c_{i}$, and $b_{i}$ represent the yield constants, maximal predator growth rates, half-saturation constants, and predator's death rates, respectively. Constants $r$ and $k$ are the prey intrinsic growth rate and carrying capacity, respectively. As observed in [13], $u_{3}$ preys on $u_{2}$ and only on $u_{2}$, and $u_{2}$ preys on $u_{1}$ and nutrient recycling is not accounted for, which produces the so-called simple food chain. A distinct feature of the simple food chain is the domino effect: if one species dies out, all the species at higher trophic levels die out as well.

As in [13], for simplicity, we use the following scaling to (2.1):

$$
\begin{aligned}
& t \rightarrow r t, \quad u_{1} \rightarrow u_{1} / k, \quad u_{2} \rightarrow c_{1} u_{2} / k, \quad u_{3} \rightarrow c_{1} c_{2} u_{3} / k, \\
& m_{1} \rightarrow m_{1} / r, \quad b_{1} \rightarrow b_{1} / r, \quad m_{2} \rightarrow m_{2} / r, \quad b_{2} \rightarrow b_{2} / r,
\end{aligned}
$$

and (2.1) becomes the form

$$
\left\{\begin{array}{rlrl}
\frac{\mathrm{d} u_{1}}{\mathrm{~d} t}=u_{1}\left(1-u_{1}\right)-\frac{a_{1} u_{1} u_{2}}{u_{1}+u_{2}}, & & t>0, \\
\frac{\mathrm{d} u_{2}}{\mathrm{~d} t}=\frac{m_{1} u_{1} u_{2}}{u_{1}+u_{2}}-b_{1} u_{2}-\frac{a_{2} u_{2} u_{3}}{u_{2}+u_{3}}, & & t>0, \\
\frac{\mathrm{d} u_{3}}{\mathrm{~d} t}=\frac{m_{2} u_{2} u_{3}}{u_{2}+u_{3}}-b_{2} u_{3}, & t>0, \\
u_{1}(0)>0, \quad u_{2}(0)>0, \quad u_{3}(0)>0, &
\end{array}\right.
$$

Copyright (c) by SIAM. Unauthorized reproduction of this article is prohibited. 
where $a_{i}=m_{i} /\left(\eta_{i} c_{i} r\right)(i=1,2)$, can be regarded as the respective predation rate of $u_{2}$ and $u_{3}$.

From [13], it is easily shown that (2.2) has a unique positive steady state solution if and only if the following are satisfied:

$$
m_{2}>b_{2}, \quad A>1 \text { and } 0<a_{1}<A /(A-1),
$$

where

$$
A \equiv m_{1} /\left(a_{2}\left(m_{2}-b_{2}\right) / m_{2}+b_{1}\right) .
$$

Moreover, the unique positive steady state $\left(u_{1}, u_{2}, u_{3}\right)=\left(\tilde{u}_{1}, \tilde{u}_{2}, \tilde{u}_{3}\right)$ can be expressed as

$$
\tilde{u}_{1}=\left[a_{1}+A\left(1-a_{1}\right)\right] / A, \quad \tilde{u}_{2}=(A-1) \tilde{u}_{1}, \quad \text { and } \quad \tilde{u}_{3}=\left(m_{2}-b_{2}\right) \tilde{u}_{2} / b_{2} .
$$

We also note that $m_{2}>b_{2}$ and $A>1$ imply $m_{1}>b_{1}$.

In [13], the authors dealt with (2.2). In particular, they obtained the extinction conditions of certain species and discussed the local asymptotical stability of $\left(\tilde{u}_{1}, \tilde{u}_{2}, \tilde{u}_{3}\right)$ and various scenarios where distinct solutions can be attracted to the origin, the pest-free steady state, and the positive steady state $\left(\tilde{u}_{1}, \tilde{u}_{2}, \tilde{u}_{3}\right)$. For more detail, we refer the reader to [13]. From their results, the authors pointed out that this ODE system is very rich in dynamics.

To take into account the inhomogeneous distribution of the predators and the prey in different spatial locations within a fixed bounded domain $\Omega$ in $\mathbf{R}^{N}$ with smooth boundary at any given time, and the natural tendency of each species to diffuse to a smaller population concentration, instead of (2.2), we need to consider the following reaction-diffusion (PDE) system:

$$
\begin{cases}u_{1 t}-d_{1} \Delta u_{1}=u_{1}\left(1-u_{1}\right)-\frac{a_{1} u_{1} u_{2}}{u_{1}+u_{2}} & \text { in } \Omega \times(0, \infty), \\ u_{2 t}-d_{2} \Delta u_{2}=\frac{m_{1} u_{1} u_{2}}{u_{1}+u_{2}}-b_{1} u_{2}-\frac{a_{2} u_{2} u_{3}}{u_{2}+u_{3}} & \text { in } \Omega \times(0, \infty), \\ u_{3 t}-d_{3} \Delta u_{3}=\frac{m_{2} u_{2} u_{3}}{u_{2}+u_{3}}-b_{2} u_{3} & \text { in } \Omega \times(0, \infty), \\ \partial_{\nu} u_{i}=0, \quad i=1,2,3, & \text { on } \partial \Omega \times(0, \infty), \\ u_{i}(x, 0)=u_{i 0}(x) \geq 0, \not \equiv 0, i=1,2,3, & \text { in } \Omega .\end{cases}
$$

Here $\nu$ is the outward unit normal vector on the boundary $\partial \Omega$ and $\partial_{\nu}=\partial / \partial \nu$, and $d_{i}(i=1,2,3)$ are called the diffusion coefficients of the corresponding species $u_{i}$ and hence are assumed to be positive constants. The initial data $u_{i 0}(i=1,2,3)$ are continuous functions, and the homogeneous Neumann boundary condition means that model (2.4) is self-contained and has no population flux across the boundary $\partial \Omega$.

In our work here, we are mainly concerned with the effect of diffusion on stationary pattern generated by (2.4). Hence, this leads us to study the steady state problem of 
(2.4), which satisfies

$$
\begin{cases}-d_{1} \Delta u_{1}=u_{1}\left(1-u_{1}\right)-\frac{a_{1} u_{1} u_{2}}{u_{1}+u_{2}} & \text { in } \Omega, \\ -d_{2} \Delta u_{2}=\frac{m_{1} u_{1} u_{2}}{u_{1}+u_{2}}-b_{1} u_{2}-\frac{a_{2} u_{2} u_{3}}{u_{2}+u_{3}} & \text { in } \Omega, \\ -d_{3} \Delta u_{3}=\frac{m_{2} u_{2} u_{3}}{u_{2}+u_{3}}-b_{2} u_{3} & \text { in } \Omega, \\ \partial_{\nu} u_{i}=0, i=1,2,3, & \text { on } \partial \Omega .\end{cases}
$$

It is evident that only nonnegative solutions of (2.5) are of real interest. The positive solution $\left(u_{1}, u_{2}, u_{3}\right)$ of $(2.5)$ to be mentioned throughout this paper always refers to a classical solution with $u_{i}>0(i=1,2,3)$ on $\bar{\Omega}$. It should also be noted that the well-known maximum principle ensures that a nonnegative classical solution of $(2.5)$ with $u_{i} \not \equiv 0(i=1,2,3)$ must be a positive one.

For (2.4) and the steady state problem (2.5), we will mainly concentrate on the coexistence of the three species and consider the case of $a_{1}<1$. In particular, some results for the existence and nonexistence of nonconstant positive solutions to (2.5) are derived. In establishing the existence of nonconstant positive solutions, due to the lack of variational structure for (2.5), our mathematical tool is the topology degree theory incorporated with the calculation of the fixed point index.

3. Persistence and stability. For simplicity of presentation, we introduce some notation. Throughout this section, let

$$
\mathbf{u}(t)=\left(u_{1}(t), u_{2}(t), u_{3}(t)\right)^{T} \quad \text { and } \quad \mathbf{u}(x, t)=\left(u_{1}(x, t), u_{2}(x, t), u_{3}(x, t)\right)^{T}
$$

be the respective solutions of (2.2) and (2.4). Denote $\mathbf{u}=\left(u_{1}, u_{2}, u_{3}\right)^{T}, \tilde{\mathbf{u}}=\left(\tilde{u}_{1}, \tilde{u}_{2}\right.$, $\left.\tilde{u}_{3}\right)^{T}$. From classical theories of ODEs and parabolic equations, $\mathbf{u}(t)$ and $\mathbf{u}(x, t)$ exist globally and are positive; namely, $u_{i}(t), u_{i}(x, t)>0(i=1,2,3)$ for all $t>0$ and $x \in \bar{\Omega}$.

First we state some simple facts about the asymptotical behavior of solutions to (2.4). The proof is similar to that of Theorem 2.5 in [28] and so is omitted here.

Proposition 3.1. The solution $\left(u_{1}(x, t), u_{2}(x, t), u_{3}(x, t)\right)$ of $(2.4)$ satisfies the following:

(i) If $m_{1} \leq b_{1}$, then $\left(u_{2}(x, t), u_{3}(x, t)\right) \rightarrow(0,0)$ uniformly on $\bar{\Omega}$ as $t \rightarrow \infty$.

(ii) If $m_{2} \leq b_{2}$, then $u_{3}(x, t) \rightarrow 0$ uniformly on $\bar{\Omega}$ as $t \rightarrow \infty$.

(iii) If $a_{1} \leq 1$ and $m_{1} \leq b_{1}$, then $u_{1}(x, t) \rightarrow 1$ and $\left(u_{2}(x, t), u_{3}(x, t)\right) \rightarrow(0,0)$ uniformly on $\bar{\Omega}$ as $t \rightarrow \infty$. As a consequence, if $m_{1} \leq b_{1}$ or $m_{2} \leq b_{2}$, problem (2.5) has no positive solutions.

As shown in Proposition 3.1, if $m_{1} \leq b_{1}$ or $m_{2} \leq b_{2}$, the two predators or the top predator will become extinct, respectively. Moreover, if $a_{1} \leq 1$ and $m_{1} \leq b_{1}$, then only the plant will exist eventually.

In this paper, since our main goal is to analyze the coexistence of the three species, from now on, unless otherwise specified, it is always assumed that $\left(\tilde{u}_{1}, \tilde{u}_{2}, \tilde{u}_{3}\right)$ exists, which implies that $m_{1}>b_{1}$ and $m_{2}>b_{2}$ as indicated in section 2 .

We have the following basic persistence property of the solutions $\mathbf{u}(t)$ and $\mathbf{u}(x, t)$, which shows that the three species always coexist at any time and any location of the habitat domain, no matter how fast or slowly they diffuse, under certain conditions on parameters. This result is even new for the ODE system (2.2). 
Proposition 3.2. Assume that $a_{1}<1, a_{2}+b_{1}<m_{1}$ hold. Then, for any $0<\varepsilon \ll 1$, there exists $T \gg 1$ such that $\mathbf{u}(t)$ and $\mathbf{u}(x, t)$ satisfy

$$
\begin{gathered}
K-\varepsilon<u_{1}(t), \quad u_{1}(x, t)<1+\varepsilon, \\
\frac{K\left(m_{1}-\left(a_{2}+b_{1}\right)\right)}{a_{2}+b_{1}}-\varepsilon<u_{2}(t), \quad u_{2}(x, t)<\frac{m_{1}-b_{1}}{b_{1}}+\varepsilon, \\
\frac{K\left(m_{1}-\left(a_{2}+b_{1}\right)\right)\left(m_{2}-b_{2}\right)}{\left(a_{2}+b_{1}\right) b_{2}}-\varepsilon<u_{3}(t), \quad u_{3}(x, t)<\frac{\left(m_{1}-b_{1}\right)\left(m_{2}-b_{2}\right)}{b_{1} b_{2}}+\varepsilon
\end{gathered}
$$

for all $x \in \bar{\Omega}$ and $t>T$. Here, $K$ is given by

$$
K=\frac{1}{2}\left\{2-\frac{m_{1}}{b_{1}}+\sqrt{\left(2-\frac{m_{1}}{b_{1}}\right)^{2}+4\left(1-a_{1}\right)\left(\frac{m_{1}}{b_{1}}-1\right)}\right\} .
$$

Proof. The proof is based on comparison principles. We first prove that the estimates hold for $\mathbf{u}(t)$. For $0<\varepsilon \ll 1$ and $t \gg 1$, from the first equation in (2.2) it is clear that $u_{1}(t)<1+\varepsilon$ by the comparison principle for ODEs.

In the following, we always consider that $0<\varepsilon \ll 1$ and $t \geq T \gg 1$, and the values of $\varepsilon$ and $T$ may be different from line to line. Since $u_{2}(t)$ satisfies

$$
u_{2}^{\prime}(t)<\frac{\left(m_{1}-b_{1}\right)(1+\varepsilon)-b_{1} u_{2}}{1+\varepsilon+u_{2}} u_{2},
$$

by the comparison principle for ODEs again, we have that

$$
u_{2}(t)<\frac{\left(m_{1}-b_{1}\right)(1+\varepsilon)}{b_{1}}+\varepsilon=\frac{m_{1}-b_{1}}{b_{1}}+\frac{m_{1}}{b_{1}} \varepsilon .
$$

Thus we can assume the following holds:

$$
u_{2}(t)<\frac{m_{1}-b_{1}}{b_{1}}+\varepsilon
$$

Combining (3.1) and the first equation in (2.2), we deduce that

$$
u_{1}^{\prime}(t)>\frac{-u_{1}^{2}+\left(2-m_{1} / b_{1}-\varepsilon\right) u_{1}+\left(1-a_{1}\right)\left[\left(m_{1}-b_{1}\right) / b_{1}+\varepsilon\right]}{\left(m_{1}-b_{1}\right) / b_{1}+\varepsilon+u_{1}} u_{1} .
$$

Therefore

$$
\begin{aligned}
u_{1}(t)> & \frac{1}{2}\left\{2-\frac{m_{1}}{b_{1}}-\varepsilon+\sqrt{\left(2-\frac{m_{1}}{b_{1}}-\varepsilon\right)^{2}+4\left(1-a_{1}\right)\left(\frac{m_{1}}{b_{1}}-1+\varepsilon\right)}\right\} \\
& -\varepsilon>K-\varepsilon .
\end{aligned}
$$

Similarly, applying (3.2) to the second equation in (2.2), we obtain

$$
u_{2}(t)>\frac{K\left(m_{1}-\left(a_{2}+b_{1}\right)\right)}{a_{2}+b_{1}}-\varepsilon .
$$

Together with (3.1) and (3.3), the third equation in (2.2) results in

$$
\frac{K\left[m_{1}-\left(a_{2}+b_{1}\right)\right]\left(m_{2}-b_{2}\right)}{\left(a_{2}+b_{1}\right) b_{2}}-\varepsilon<u_{3}(t)<\frac{\left(m_{1}-b_{1}\right)\left(m_{2}-b_{2}\right)}{b_{1} b_{2}}+\varepsilon .
$$

Copyright (c) by SIAM. Unauthorized reproduction of this article is prohibited. 
To sum up, (3.1)-(3.4) deduce our result for $\mathbf{u}(t)$. In a similar manner, by the comparison principle for parabolic equations, one can establish the desired estimates for $\mathbf{u}(x, t)$.

In particular Proposition 3.2 and the maximum principle imply a priori upper and lower bounds for the positive solutions of (2.5), which will play crucial roles in the later sections. To prove that we recall the following maximum principle (for example, Lemma 2.1 in [21]).

Lemma 3.1. Suppose that $g \in C(\bar{\Omega} \times \mathbf{R})$.

(i) Assume that $w \in C^{2}(\Omega) \cap C^{1}(\bar{\Omega})$ and satisfies

$$
\Delta w(x)+g(x, w(x)) \geq 0 \text { in } \Omega, \quad \partial_{\nu} w \leq 0 \text { on } \partial \Omega .
$$

If $w\left(x_{0}\right)=\max _{\bar{\Omega}} w$, then $g\left(x_{0}, w\left(x_{0}\right)\right) \geq 0$.

(ii) Assume that $w \in C^{2}(\Omega) \cap C^{1}(\bar{\Omega})$ and satisfies

$$
\Delta w(x)+g(x, w(x)) \leq 0 \text { in } \Omega, \quad \partial_{\nu} w \geq 0 \text { on } \partial \Omega .
$$

If $w\left(x_{0}\right)=\min _{\bar{\Omega}} w$, then $g\left(x_{0}, w\left(x_{0}\right)\right) \leq 0$.

Now we have the following a priori estimates for steady state solutions.

TheOrem 3.1. Assume that $a_{1}<1$ and $a_{2}+b_{1}<m_{1}$ hold. Let $K$ be defined as in Proposition 3.2. Then any positive solution $\left(u_{1}, u_{2}, u_{3}\right)$ of $(2.5)$ satisfies the following: for all $x \in \bar{\Omega}$,

$$
\begin{gathered}
K<u_{1}(x)<1, \\
\frac{K\left(m_{1}-\left(a_{2}+b_{1}\right)\right)}{a_{2}+b_{1}}<u_{2}(x)<\frac{m_{1}-b_{1}}{b_{1}}, \\
\frac{K\left(m_{1}-\left(a_{2}+b_{1}\right)\right)\left(m_{2}-b_{2}\right)}{\left(a_{2}+b_{1}\right) b_{2}}<u_{3}(x)<\frac{\left(m_{1}-b_{1}\right)\left(m_{2}-b_{2}\right)}{b_{1} b_{2}} .
\end{gathered}
$$

Proof. From Proposition 3.2, stated results hold if strict inequalities are replaced by nonstrict inequalities. Thus we only need to show the strict inequalities. Let $\left(u_{1}, u_{2}, u_{3}\right)$ be a positive solution of $(2.5)$ and set

$$
u_{i}\left(x_{i}\right)=\max _{\bar{\Omega}} u_{i} \quad \text { and } \quad u_{i}\left(y_{i}\right)=\min _{\bar{\Omega}} u_{i}, \quad i=1,2,3 .
$$

Applying Lemma 3.1 to the first equation in (2.5), we find that

$$
1-u_{1}\left(x_{1}\right)-\frac{a_{1} u_{2}\left(x_{1}\right)}{u_{1}\left(x_{1}\right)+u_{2}\left(x_{1}\right)} \geq 0 .
$$

Thus when $a_{1}<1$, it follows that $u_{1}\left(y_{1}\right)<1$. Following the same order in the proof of Proposition 3.2, we can show that the stated results with strict inequalities hold.

When the population persistence holds for the food chain, the constant steady state $\tilde{\mathbf{u}}$ is always in the attracting region given in Proposition 3.2 and Theorem 3.1. Next we discuss the stability of $\tilde{\mathbf{u}}$ with respect to (2.4). To this end, we need to collect some known facts from [13]. For sake of simplicity, we denote

$$
\mathbf{G}(\mathbf{u})=\left(\begin{array}{c}
u_{1}\left(1-u_{1}\right)-\frac{a_{1} u_{1} u_{2}}{u_{1}+u_{2}} \\
\frac{m_{1} u_{1} u_{2}}{u_{1}+u_{2}}-b_{1} u_{2}-\frac{a_{2} u_{2} u_{3}}{u_{2}+u_{3}} \\
\frac{m_{2} u_{2} u_{3}}{u_{2}+u_{3}}-b_{2} u_{3}
\end{array}\right) \quad \text { and } \quad \mathbf{G}_{\mathbf{u}}(\tilde{\mathbf{u}})=\left(\begin{array}{ccc}
a_{11} & a_{12} & 0 \\
a_{21} & a_{22} & a_{23} \\
0 & a_{32} & a_{33}
\end{array}\right) \text {, }
$$

Copyright (c) by SIAM. Unauthorized reproduction of this article is prohibited. 
where

$$
\left\{\begin{array}{l}
a_{11}=\tilde{u}_{1}\left[-1+\frac{a_{1} \tilde{u}_{2}}{\left(\tilde{u}_{1}+\tilde{u}_{2}\right)^{2}}\right], \quad a_{22}=\tilde{u}_{2}\left[-\frac{m_{1} \tilde{u}_{1}}{\left(\tilde{u}_{1}+\tilde{u}_{2}\right)^{2}}+\frac{a_{2} \tilde{u}_{3}}{\left(\tilde{u}_{2}+\tilde{u}_{3}\right)^{2}}\right], \\
a_{33}=-\frac{m_{2} \tilde{u}_{2} \tilde{u}_{3}}{\left(\tilde{u}_{2}+\tilde{u}_{3}\right)^{2}}<0, \quad a_{12}=-\frac{a_{1} \tilde{u}_{1}^{2}}{\left(\tilde{u}_{1}+\tilde{u}_{2}\right)^{2}}<0, \quad a_{21}=\frac{m_{1} \tilde{u}_{2}^{2}}{\left(\tilde{u}_{1}+\tilde{u}_{2}\right)^{2}}>0, \\
a_{23}=-a_{2} \tilde{u}_{2}^{2} /\left(\tilde{u}_{2}+\tilde{u}_{3}\right)^{2}<0, \quad a_{32}=m_{2} \tilde{u}_{3}^{2} /\left(\tilde{u}_{2}+\tilde{u}_{3}\right)^{2}>0 .
\end{array}\right.
$$

In Proposition 3.1 in [13], it was proved that if $a_{11} \leq 0$ and $a_{22} \leq 0$, $\tilde{\mathbf{u}}$ is locally asymptotically stable for (2.2). Indeed even with the presence of the diffusion, $\tilde{\mathbf{u}}$ is uniformly asymptotically stable for (2.4) under the same conditions. More precisely, we have the following theorem.

THEOREM 3.2. Assume that $a_{11} \leq 0$ and $a_{22} \leq 0$ hold; then $\tilde{\mathbf{u}}$ is locally uniformly asymptotically stable for (2.4) in the sense of [12]. As a consequence, (2.5) has no nonconstant positive solution in a neighborhood of $\tilde{\mathbf{u}}$. Moreover, if $a_{1}<1$ and $a_{2}+b_{1}<$ $m_{1}$, then $a_{11}<0$ and $a_{22}<0 ;$ hence $\tilde{\mathbf{u}}$ is locally uniformly asymptotically stable.

Proof. The proof of stability when $a_{11} \leq 0$ and $a_{22} \leq 0$ is similar to that of Theorem 2 in [36], and we omit the details here. We note that if $a_{1}<1$, then $a_{11}<0$. Moreover, the inequality $a_{22} \leq 0$ is equivalent to

$$
m_{1} \geq\left(b_{1}+a_{2} \frac{m_{2}-b_{2}}{m_{2}}\right)^{2} /\left(b_{1}+a_{2}\left(\frac{m_{2}-b_{2}}{m_{2}}\right)^{2}\right) .
$$

It is also noted that

$$
\left(b_{1}+a_{2} \frac{m_{2}-b_{2}}{m_{2}}\right)^{2} /\left(b_{1}+a_{2}\left(\frac{m_{2}-b_{2}}{m_{2}}\right)^{2}\right)<a_{2}+b_{1} .
$$

Hence if $a_{1}<1$ and $a_{2}+b_{1}<m_{1}$, we have $a_{11}, a_{22}<0$ by (3.5).

Remark 3.1. Theorem 3.2 and the previous arguments show that no Turing instability or diffusion-driven instability phenomenon occurs when $a_{11} \leq 0$ and $a_{22} \leq 0$ hold. On the other hand, if we take $m_{1}=\left(b_{1}+a_{2}\left(m_{2}-b_{2}\right) / m_{2}\right)^{2} /\left(b_{1}+a_{2}\left(m_{2}-\right.\right.$ $\left.\left.b_{2}\right)^{2} / m_{2}^{2}\right)-o\left(b_{1}\right)$, and $b_{1} \rightarrow 0, a_{2}, b_{2}, m_{2}$ are properly chosen and either $a_{1} \rightarrow 1 / 2$ or $a_{1} \rightarrow 1$, as in Proposition 3.1 in [13], together with some meticulous computations, the well-known Roth-Hurwitz criterion ensures that $\tilde{\mathbf{u}}$ is still stable for the ODE system (2.2). However, by fixing these parameters including $d_{1}$ and $d_{2}$, and then letting the diffusion $d_{3}$ be large enough, similar to the proof of Theorem 2 in [36], one can show that $\tilde{\mathbf{u}}$ is unstable with respect to the PDE system (2.4). Thus Turing instability could occur when the conditions of Theorem 3.2 are not satisfied.

From Theorem 3.2, $\tilde{\mathbf{u}}$ is locally uniformly asymptotically stable when $a_{1}<1$ and $a_{2}+b_{1}<m_{1}$. In this case it is unlikely that nonconstant positive solutions (stationary pattern) of (2.5) exist. Indeed with more restrictive conditions on the parameters, we can show the global stability of $\tilde{\mathbf{u}}$ for systems (2.2) and (2.4). Our result below is independent of the diffusion rates $d_{i}$; that is, the constant coexistence state $\tilde{\mathbf{u}}$ is globally asymptotically stable. Hence when the conditions on the parameters are satisfied, $\tilde{\mathbf{u}}$ is stabilized under arbitrary spatially inhomogeneous perturbation.

Theorem 3.3. Let $K$ be defined as in Proposition 3.2. Assume that the following hold:

(i) $a_{1}<1$ and $a_{2}+b_{1}<m_{1}$;

(ii) $a_{1}(A-1) / A<m_{1} K /\left(a_{2}+b_{1}\right)$; 
(iii) $a_{2} b_{2} m_{1}\left(m_{2}-b_{2}\right)\left(a_{2}+b_{1}\right)<b_{1} m_{2} K\left[m_{1}-\left(a_{2}+b_{1}\right)\right]\left[b_{1} m_{2}+a_{2}\left(m_{2}-b_{2}\right)\right]$.

Then the constant positive steady state $\tilde{\mathbf{u}}$ is globally asymptotically stable for systems (2.2) and (2.4) for all initial nonnegative conditions which are not steady states. In particular, (2.5) has no nonconstant positive solution if conditions (i)-(iii) hold.

Proof. We use Lyapunov functionals for the proof. First, we verify the result for system (2.2). For our purpose, we first recall the following basic Lyapunov functionals:

$$
E\left(u_{i}\right)=u_{i}-\tilde{u}_{i}-\tilde{u}_{i} \ln \frac{u_{i}}{\tilde{u}_{i}}, \quad i=1,2,3 .
$$

Note that $E\left(u_{i}(t)\right)$ are nonnegative, and $E\left(u_{i}(t)\right)=0(i=1,2,3)$ if and only if $\left(u_{1}(t), u_{2}(t), u_{3}(t)\right)=\left(\tilde{u}_{1}, \tilde{u}_{2}, \tilde{u}_{3}\right)$. Hence, letting

$$
E(t)=E\left(u_{1}(t)\right)+\frac{a_{1} \tilde{u}_{1}}{m_{1} \tilde{u}_{2}} E\left(u_{2}(t)\right)+\frac{a_{1} a_{2} \tilde{u}_{1}}{m_{1} m_{2} \tilde{u}_{3}} E\left(u_{3}(t)\right),
$$

we have

$$
\begin{aligned}
\frac{d E}{d t}=\{ & \left.-1+\frac{a_{1} \tilde{u}_{2}}{\left(\tilde{u}_{1}+\tilde{u}_{2}\right)\left(u_{1}+u_{2}\right)}\right\}\left(u_{1}-\tilde{u}_{1}\right)^{2}+\frac{a_{1} \tilde{u}_{1}}{m_{1} \tilde{u}_{2}}\left\{-\frac{m_{1} \tilde{u}_{1}}{\left(\tilde{u}_{1}+\tilde{u}_{2}\right)\left(u_{1}+u_{2}\right)}\right. \\
& \left.+\frac{a_{2} \tilde{u}_{3}}{\left(\tilde{u}_{2}+\tilde{u}_{3}\right)\left(u_{2}+u_{3}\right)}\right\}\left(u_{2}-\tilde{u}_{2}\right)^{2}-\frac{a_{1} a_{2} \tilde{u}_{1} \tilde{u}_{2}}{m_{1} \tilde{u}_{3}\left(\tilde{u}_{2}+\tilde{u}_{3}\right)\left(u_{2}+u_{3}\right)}\left(u_{3}-\tilde{u}_{3}\right)^{2} .
\end{aligned}
$$

Under our assumptions (i)-(iii), we can claim that for $t \gg 1$ the following hold:

$$
\frac{a_{1} \tilde{u}_{2}}{\left(\tilde{u}_{1}+\tilde{u}_{2}\right)\left(u_{1}+u_{2}\right)} \leq 1 \text { and } \frac{a_{2} \tilde{u}_{3}}{\left(\tilde{u}_{2}+\tilde{u}_{3}\right)\left(u_{2}+u_{3}\right)} \leq \frac{m_{1} \tilde{u}_{1}}{\left(\tilde{u}_{1}+\tilde{u}_{2}\right)\left(u_{1}+u_{2}\right)} .
$$

In fact, by Proposition 3.2, to satisfy (3.7), for $t \gg 1$ it is sufficient to require

$$
\frac{a_{1} \tilde{u}_{2}}{\left(\tilde{u}_{1}+\tilde{u}_{2}\right)}<\frac{m_{1} K}{a_{2}+b_{1}} \text { and } \frac{a_{2} \tilde{u}_{3}}{\left(\tilde{u}_{2}+\tilde{u}_{3}\right)} \cdot \frac{m_{1}}{b_{1}}<\frac{m_{1} \tilde{u}_{1}}{\left(\tilde{u}_{1}+\tilde{u}_{2}\right)} \cdot \frac{K m_{2}\left(m_{1}-\left(a_{2}+b_{1}\right)\right)}{\left(a_{2}+b_{1}\right) b_{2}} \text {. }
$$

Therefore by the definition of $\left(\tilde{u}_{1}, \tilde{u}_{2}, \tilde{u}_{3}\right)$, we easily see that the above two inequalities are equivalent to assumptions (ii) and (iii), respectively. Thus (3.6) implies that $E^{\prime}(t)<0$ for $t \gg 1$. Now for $t \gg 1, E(t)$ is a Lyapunov functional for system (2.2); namely, for $t \gg 1, E^{\prime}(t)<0$ along trajectories and $E(t)>0$ except at $\tilde{\mathbf{u}}$. Hence $\tilde{\mathbf{u}}$ is globally asymptotically stable for (2.2) following the well-known theorem of Lyapunov stability.

Based on the proof of Theorem 3.3, by Proposition 3.2, it is not hard to see that for $t \gg 1$

$$
E^{*}(t)=\int_{\Omega}\left\{E\left(u_{1}(x, t)\right)+\frac{a_{1} \tilde{u}_{1}}{m_{1} \tilde{u}_{2}} E\left(u_{2}(x, t)\right)+\frac{a_{1} a_{2} \tilde{u}_{1}}{m_{1} m_{2} \tilde{u}_{3}} E\left(u_{3}(x, t)\right)\right\} \mathrm{d} x
$$

is a Lyapunov functional for system (2.4) and $\tilde{\mathbf{u}}$ is globally asymptotically stable for system (2.4) under our assumptions.

Remark 3.2. Simple analysis shows that Theorem 3.3 holds if one of the following holds: (1) $a_{1} \rightarrow 0, a_{2} \rightarrow 0$; (2) $a_{1}<1, m_{1}$ is large and $m_{2} \rightarrow b_{2}$; or (3) $a_{1}<1, m_{1}$ is large, and $a_{2} b_{2}\left(m_{2}-b_{2}\right)\left(a_{2}+b_{1}\right)<\left(1-a_{1}\right) b_{1} m_{2}\left[b_{1} m_{2}+a_{2}\left(m_{2}-b_{2}\right)\right]$. Indeed in case (1), $K$ defined in Proposition 3.2 tends to 1 as $a_{1} \rightarrow 0$, and the lower and upper bounds in Proposition 3.2 and Theorem 3.1 tend to the same value as $a_{2} \rightarrow 0$.

Copyright $@$ by SIAM. Unauthorized reproduction of this article is prohibited. 
This shows that the a priori estimate in Proposition 3.2 and Theorem 3.1 are sharp when $a_{1}$ and $a_{2}$ are small.

Results in this section have interesting and significant biological implications. Regarding the impact of the diffusion, all results in this section (Propositions 3.1 and 3.2 and Theorems 3.1, 3.2, and 3.3) are independent of diffusion coefficients $d_{i}, i=1,2,3$. In these parameter ranges, diffusion usually enhances the stability of the constant steady states. Proposition 3.1 gives conditions of total extinction of all three species and conditions of the extinction of both middle and top predators. Comparison can be made with results in section 2 of [13], where the ODE system is studied in more detail.

When the constant coexistence steady state $\tilde{\mathbf{u}}$ exists, our main persistence and stability results are proved under the assumptions

$$
a_{1}<1 \text { and } a_{2}+b_{1}<m_{1} .
$$

These conditions are evidently stronger than the conditions (2.3) under which $\tilde{\mathbf{u}}$ exists. But with (3.8) satisfied, persistence holds for the whole food chain, and all three species coexist regardless of initial conditions (see Proposition 3.2). The persistence question is even open for the same ODE system, and here we prove it for the more general reaction-diffusion system with no-flux boundary condition. This answers an open question raised in [13] (see discussion on p. 80). Moreover, under (3.8), $\tilde{\mathbf{u}}$ is also locally uniformly asymptotically stable with respect to (2.4), and under strong conditions in Theorem 3.3, ũ is globally asymptotically stable. For the ODE systems, these results complement those in [13] in which the main concern is successful biological control. Indeed our results show that under (3.8), biological control of the pest cannot be achieved.

4. Nonexistence of nonconstant positive solutions of (2.5). In Theorem 3.3, the global stability of the constant coexistence steady state implies the nonexistence of nonconstant positive solutions of (2.5) regardless of diffusions. Several nonexistence results of nonconstant positive solutions to (2.5) will be presented in this section, and in these results, the diffusion coefficients do play important roles. The mathematical techniques to be employed are the implicit function theorem method and the energy method, respectively. From now on, let $0=\mu_{0}<\mu_{1} \leq \mu_{2} \leq \cdots$ be the eigenvalues of the operator $-\Delta$ on $\Omega$ with the homogeneous Neumann boundary condition.

4.1. The energy method. In this subsection, we apply the energy method to establish some results on the nonexistence of nonconstant positive solutions of (2.5). For convenience, let us denote the constants $a_{i}, b_{i}, m_{i}(i=1,2)$ collectively by $\Lambda$.

TheOREM 4.1. Assume that $a_{1}<1$ and $a_{2}+b_{1}<m_{1}$.

(i) There exists $\hat{D}_{1,2}=\hat{D}_{1,2}(\Lambda)$ which is independent of $d_{3}$ and $\Omega$, such that (2.5) has no nonconstant positive solution provided that $\min \left\{\mu_{1} d_{1}, \mu_{1} d_{2}\right\} \geq \hat{D}_{1,2}$.

(ii) If, in addition, $a_{1}\left(a_{2}+b_{1}\right)^{2}\left(m_{1}-b_{1}\right) \leq\left(1-a_{1}\right)^{2} b_{1} m_{1}^{2}$, then there exists $\hat{D}_{2}=\hat{D}_{2}(\Lambda)$ which is independent of $d_{1}, d_{3}$, and $\Omega$, such that (2.5) has no nonconstant positive solution provided that $\mu_{1} d_{2} \geq \hat{D}_{2}$.

(iii) If, in addition, $a_{2}\left(a_{2}+b_{1}\right)^{2} b_{2} m_{1}\left(m_{1}-b_{1}\right)\left(m_{2}-b_{2}\right) \leq\left(1-a_{1}\right)^{3} b_{1}^{3} m_{2}^{2}\left(m_{1}-\right.$ $\left.\left(a_{2}+b_{1}\right)\right)^{2}$, then there exists $\hat{D}_{1,3}=\hat{D}_{1,3}(\Lambda)$ which is independent of $d_{2}$ and $\Omega$, such that (2.5) has no nonconstant positive solution provided that $\min \left\{\mu_{1} d_{1}, \mu_{1} d_{3}\right\} \geq \hat{D}_{1,3}$. 
Proof. Let $\left(u_{1}, u_{2}, u_{3}\right)$ be a positive solution of $(2.5)$ and let $\bar{g}=|\Omega|^{-1} \int_{\Omega} g \mathrm{dx}$. Then, multiplying the corresponding equation in $(2.5)$ by $\frac{1}{u_{i}}\left(u_{i}-\bar{u}_{i}\right), i=1,2,3$, integrating over $\Omega$, and adding the results, we get

$$
\begin{aligned}
\int_{\Omega}\left\{\sum_{i=1}^{3} \frac{d_{i} \bar{u}_{i}\left|\nabla\left(u_{i}-\bar{u}_{i}\right)\right|^{2}}{u_{i}^{2}}\right\} \mathrm{d} x \\
\quad=\int_{\Omega}\left\{\left(u_{1}-\bar{u}_{1}\right)^{2}\left(-1+\frac{a_{1} \bar{u}_{2}}{\left(u_{1}+u_{2}\right)\left(\bar{u}_{1}+\bar{u}_{2}\right)}\right)\right. \\
\quad+\left(u_{1}-\bar{u}_{1}\right)\left(u_{2}-\bar{u}_{2}\right) \frac{-a_{1} \bar{u}_{1}+m_{1} \bar{u}_{2}}{\left(u_{1}+u_{2}\right)\left(\bar{u}_{1}+\bar{u}_{2}\right)} \\
\quad+\left(u_{2}-\bar{u}_{2}\right)^{2}\left(\frac{-m_{1} \bar{u}_{1}}{\left(u_{1}+u_{2}\right)\left(\bar{u}_{1}+\bar{u}_{2}\right)}+\frac{a_{2} \bar{u}_{3}}{\left(u_{2}+u_{3}\right)\left(\bar{u}_{2}+\bar{u}_{3}\right)}\right) \\
\left.\quad+\left(u_{2}-\bar{u}_{2}\right)\left(u_{3}-\bar{u}_{3}\right) \frac{-a_{2} \bar{u}_{2}+m_{2} \bar{u}_{3}}{\left(u_{2}+u_{3}\right)\left(\bar{u}_{2}+\bar{u}_{3}\right)}-\left(u_{3}-\bar{u}_{3}\right)^{2} \frac{m_{2} \bar{u}_{2}}{\left(u_{2}+u_{3}\right)\left(\bar{u}_{2}+\bar{u}_{3}\right)}\right\} \mathrm{d} x .
\end{aligned}
$$

By Theorem 3.1 and the Young inequality, from (4.1) it follows that

$$
\begin{aligned}
\int_{\Omega} \sum_{i=1}^{3} d_{i}\left|\nabla\left(u_{i}-\bar{u}_{i}\right)\right|^{2} \mathrm{~d} x \leq & C \int_{\Omega}\left\{\left(u_{1}-\bar{u}_{1}\right)^{2}\left(-1+\frac{a_{1} \bar{u}_{2}}{\left(u_{1}+u_{2}\right)\left(\bar{u}_{1}+\bar{u}_{2}\right)}+\varepsilon\right)\right. \\
& +C(\varepsilon)\left(u_{2}-\bar{u}_{2}\right)^{2} \\
& \left.+\left(u_{3}-\bar{u}_{3}\right)^{2}\left(-\frac{m_{2} \bar{u}_{2}}{\left(u_{2}+u_{3}\right)\left(\bar{u}_{2}+\bar{u}_{3}\right)}+\varepsilon\right)\right\} \mathrm{d} x .
\end{aligned}
$$

Here, $C$ depends only on $\Lambda$, and $C(\varepsilon)$ depends only on $\Lambda$ and $\varepsilon$. By Theorem 3.1 again, we can choose $0<\varepsilon \ll 1$ which depends only on $\Lambda$ such that

$$
-\frac{m_{2} \bar{u}_{2}}{\left(u_{2}+u_{3}\right)\left(\bar{u}_{2}+\bar{u}_{3}\right)}+\varepsilon<0 .
$$

Thus, with (4.2) and the Poincaré inequality,

$$
\mu_{1} \int_{\Omega}(g-\bar{g})^{2} \mathrm{~d} x \leq \int_{\Omega}|\nabla(g-\bar{g})|^{2} \mathrm{~d} x,
$$

we find that

$$
\mu_{1} \int_{\Omega} \sum_{i=1}^{3} d_{i}\left(u_{i}-\bar{u}_{i}\right)^{2} \mathrm{dx} \leq C(\varepsilon) \int_{\Omega} \sum_{i=1}^{2}\left(u_{i}-\bar{u}_{i}\right)^{2} \mathrm{~d} x .
$$

By the above inequality, it is clear that there exists $\hat{D}_{1,2}$ depending only on $\Lambda$, such that when $\min \left\{\mu_{1} d_{1}, \mu_{1} d_{2}\right\} \geq \hat{D}_{1,2}, u_{i} \equiv \bar{u}_{i}=$ constant, $i=1,2,3$, which asserts our result (i). implies

If, in addition, we assume $a_{1}\left(a_{2}+b_{1}\right)^{2}\left(m_{1}-b_{1}\right) \leq\left(1-a_{1}\right)^{2} b_{1} m_{1}^{2}$, then Theorem 3.1

$$
-1+\frac{a_{1} \bar{u}_{2}}{\left(u_{1}+u_{2}\right)\left(\bar{u}_{1}+\bar{u}_{2}\right)}<0
$$

Copyright $@$ by SIAM. Unauthorized reproduction of this article is prohibited. 
Therefore, for $0<\varepsilon \ll 1$ satisfying

$$
-1+\frac{a_{1} \bar{u}_{2}}{\left(u_{1}+u_{2}\right)\left(\bar{u}_{1}+\bar{u}_{2}\right)}+\varepsilon<0 \text { and }-\frac{m_{2} \bar{u}_{2}}{\left(u_{2}+u_{3}\right)\left(\bar{u}_{2}+\bar{u}_{3}\right)}+\varepsilon<0,
$$

as before, (4.2) implies

$$
\int_{\Omega} \sum_{i=1}^{3} d_{i}\left|\nabla\left(u_{i}-\bar{u}_{i}\right)\right|^{2} \mathrm{~d} x \leq C(\varepsilon) \int_{\Omega}\left(u_{2}-\bar{u}_{2}\right)^{2} \mathrm{~d} x .
$$

Similar to arguments above, from (4.3) and the Poincaré inequality, there exists $\hat{D}_{2}=$ $\hat{D}_{2}(\Lambda)$ such that $(2.5)$ has no nonconstant positive solution if $\mu_{1} d_{2}>\hat{D}_{2}$. Thus (ii) holds.

To prove (iii), as in the arguments above, it is enough to verify that

$$
a_{2} \bar{u}_{3}\left(u_{1}+u_{2}\right)\left(\bar{u}_{1}+\bar{u}_{2}\right)<m_{1} \bar{u}_{1}\left(u_{2}+u_{3}\right)\left(\bar{u}_{2}+\bar{u}_{3}\right) .
$$

By Theorem 3.1 again, to ensure (4.4), it suffices to require that the third condition in (iii) holds. This completes our proof.

THEOREM 4.2 .

(i) Let $d_{1}^{*}, d_{3}^{*}$ be fixed positive constants satisfying $\mu_{1} d_{1}^{*}>1$ and $\mu_{1} d_{3}^{*}>m_{2}-b_{2}$. Then there exists a positive constant $D_{2}^{*}=D_{2}^{*}\left(d_{1}^{*}, d_{3}^{*}, \Lambda\right)$ such that (2.5) has no nonconstant positive solution provided that $\mu_{1} d_{2} \geq D_{2}^{*}, d_{1} \geq d_{1}^{*}$, and $d_{3} \geq d_{3}^{*}$.

(ii) Let $d_{2}^{*}$ be a fixed positive constant satisfying $\mu_{1} d_{2}^{*}>m_{1}-b_{1}$. Then there exists a positive constant $D_{1,3}^{*}=D_{1,3}^{*}\left(d_{2}^{*}, \Lambda\right)$ such that $(2.5)$ has no nonconstant positive solution provided that $\min \left\{\mu_{1} d_{1}, \mu_{1} d_{3}\right\} \geq D_{1,3}^{*}$ and $d_{2} \geq d_{2}^{*}$.

Proof. We prove only (i), and the verification of (ii) is similar. Suppose that $\left(u_{1}, u_{2}, u_{3}\right)$ and $\left(\bar{u}_{1}, \bar{u}_{2}, \bar{u}_{3}\right)$ are the same as in the proof of Theorem 4.1. Multiplying the corresponding equation of (2.5) by $u_{i}-\bar{u}_{i}, i=1,2,3$, the analysis similar to the proof of Theorem 4.1 deduces

$$
\begin{aligned}
\mu_{1} \sum_{i=1}^{3} \int_{\Omega} d_{i}\left(u_{i}-\bar{u}_{i}\right)^{2} \mathrm{~d} x \leq & \int_{\Omega}\left\{(1+\varepsilon)\left(u_{1}-\bar{u}_{1}\right)^{2}\right. \\
& \left.+C\left(u_{2}-\bar{u}_{2}\right)^{2}+\left(m_{2}-b_{2}+\varepsilon\right)\left(u_{3}-\bar{u}_{3}\right)^{2}\right\} \mathrm{d} x
\end{aligned}
$$

for some positive constant $C=C(\Lambda, \varepsilon)$. Choose $\varepsilon>0$ to be so small that $d_{1} \mu_{1} \geq$ $1+\varepsilon, d_{3} \mu_{1} \geq m_{2}-b_{2}+\varepsilon$; then there exists $D_{2}^{*}$ such that $\left(u_{1}, u_{2}, u_{3}\right)=\left(\bar{u}_{1}, \bar{u}_{2}, \bar{u}_{3}\right)$ must hold if $d_{2} \geq D_{2}^{*}$, and so our conclusion holds.

The results in this subsection demonstrate such a phenomenon: when all diffusion coefficients are large, no patterns exist. Here either $d_{1}, d_{3}$, or $d_{2}$ has a lower bound (see Theorem 4.2). If, in addition, the conditions (3.8) are satisfied, then the patterns do not exist even if only one or two diffusion coefficients are large. Such results for general reaction-diffusion systems appeared in [6], and our results here show more delicate dependence on the diffusion coefficients only for the food chain system (2.4) and (2.5).

4.2. The implicit function theorem method. In this subsection, we use the implicit function theorem to obtain some further results for the nonexistence of nonconstant positive solutions of (2.5). We will need the following a priori estimate. 
TheOREM 4.3. Let $a_{1}<1$ and let $d$ be a fixed positive number. Assume that for any positive constants $\tilde{d}_{2}$ and $\tilde{d}_{3}$, the boundary value problem

$$
\begin{cases}-\tilde{d}_{2} \Delta w_{2}=\left(m_{1}-b_{1}\right) w_{2}-\frac{a_{2} w_{2} w_{3}}{w_{2}+w_{3}} & \text { in } \Omega, \\ -\tilde{d}_{3} \Delta w_{3}=\frac{m_{2} w_{2} w_{3}}{w_{2}+w_{3}}-b_{2} w_{3} & \text { in } \Omega, \\ \partial_{\nu} w_{2}=\partial_{\nu} w_{3}=0 & \text { on } \partial \Omega\end{cases}
$$

has no positive solution satisfying $\left|w_{2}\right|_{\infty}+\left|w_{3}\right|_{\infty}=1$. Then there exist positive constants $C_{1}(\Lambda, \Omega, d)$ and $C_{2}(\Lambda, \Omega, d)$ such that any positive solution $\left(u_{1}, u_{2}, u_{3}\right)$ of (2.5) satisfies

$$
C_{1}(\Lambda, \Omega, d) \leq u_{i} \leq C_{2}(\Lambda, \Omega, d), \quad i=1,2,3,
$$

provided that $d_{1}, d_{2}, d_{3} \geq d$.

Proof. Since $a_{1}<1$, from the proof of Theorem 3.1, we see that

$$
1-a_{1}<u_{1}<1, \quad u_{2}<\left(m_{1}-b_{1}\right) / b_{1}, \quad \text { and } u_{3}<\left(m_{1}-b_{1}\right)\left(m_{2}-b_{2}\right) /\left(b_{1} b_{2}\right),
$$

so $C_{2}(\Lambda, \Omega, d)$ has been found. Similarly to the proof of Theorem 3.4 in [28], from the second and third equations in $(2.5)$, the desired $C_{1}(\Lambda, \Omega, d)$ can be obtained.

The assumption that (4.5) has no positive solution is satisfied in some important parameter ranges.

Lemma 4.1. Problem (4.5) has no positive solution if one of the following holds:

(i) $a_{2}+b_{1} \leq m_{1}$; or

(ii) $a_{2}+b_{1}>m_{1}$ and $\sqrt{a_{2}+m_{2}}<\sqrt{m_{1}-b_{1}}+\sqrt{b_{2}}$.

In particular, if $a_{1}<1$ and either (i) or (ii) holds, the a priori estimate in Theorem 4.3 holds.

Proof. If condition (i) holds, our conclusion is derived from (ii) of Lemma 3.1; if condition (ii) is satisfied, the proof is the same as that of Corollary 3.5 in [28].

In this subsection, we will prove a result which considerably improves Theorem 4.2 if the estimates in Theorem 4.3 hold. We note that the conditions (i) and (ii) include (3.8); thus the results are along the same lines as those in the last subsection. To prove our result, we first prepare two lemmas.

Lemma 4.2. Assume that $f(u)$ is a continuous function in $[0, \infty)$ and for some positive constant $a, f(u)>0$ in $(0, a)$ and $f(u)<0$ in $(a, \infty)$. Then the problem

$$
-\Delta u=u f(u) \text { in } \Omega, \quad \partial_{\nu} u=0 \text { on } \partial \Omega
$$

has a unique positive solution $u(x) \equiv a$.

Proof. The above result is easily obtained by the direct application of Lemma 3.1.

Lemma 4.3. (i) Assume that $a_{1}<1$ and that assumptions in Theorem 4.3 hold. Let $\left(u_{1 i}, u_{2 i}, u_{3 i}\right)$ be a sequence of positive solutions of $(2.5)$ with $d_{2}=d_{2 i}$ and $d_{2 i} \rightarrow \infty$ as $i \rightarrow \infty$. Then $\left(u_{1 i}, u_{2 i}, u_{3 i}\right)$ converges to $\tilde{\mathbf{u}}$ in $[C(\bar{\Omega})]^{3}$ as $i \rightarrow \infty$.

(ii) Assume that $a_{1}<1$ and that assumptions in Theorem 4.3 hold. Let $\left(u_{1 i}, u_{2 i}, u_{3 i}\right)$ be a sequence of positive solutions of $(2.5)$ with $\left(d_{1}, d_{3}\right)=\left(d_{1 i}, d_{3 i}\right)$ and $d_{1 i}, d_{3 i} \rightarrow \infty$ as $i \rightarrow \infty$. Then $\left(u_{1 i}, u_{2 i}, u_{3 i}\right)$ converges to $\tilde{\mathbf{u}}$ in $[C(\bar{\Omega})]^{3}$ as $i \rightarrow \infty$.

Proof. We prove only (i), and (ii) can be proved similarly by using Theorem 3.1 and Lemma 3.1. 
From Theorem 4.3, the sequence $\left\{\left(u_{1 i}, u_{2 i}, u_{3 i}\right)\right\}$ is bounded in $[C(\bar{\Omega})]^{3}$ with the bound independent of $d_{2}$. Then some standard arguments show that there is a subsequence of $\left(u_{1 i}, u_{2 i}, u_{3 i}\right)$ (still labelled by itself), such that $\left(u_{1 i}, u_{2 i}, u_{3 i}\right) \rightarrow\left(u_{1}, u_{2}, u_{3}\right)$ in $[C(\bar{\Omega})]^{3}$ as $i \rightarrow \infty$. Furthermore, $u_{2} \equiv c$, which is a positive constant; $u_{1}, u_{3}>0$ on $\bar{\Omega}$; and $\left(u_{1}, c, u_{3}\right)$ solves

$$
\begin{cases}-d_{1} \Delta u_{1}=u_{1}\left(1-u_{1}\right)-\frac{a_{1} c u_{1}}{u_{1}+c} \quad \text { in } \Omega, & \partial_{\nu} u_{1}=0 \text { on } \partial \Omega, \\ \int_{\Omega}\left\{\frac{m_{1} u_{1}}{u_{1}+c}-b_{1}-\frac{a_{2} u_{3}}{c+u_{3}}\right\} \mathrm{d} x=0, & \text { in } \Omega, \quad \partial_{\nu} u_{3}=0 \text { on } \partial \Omega . \\ -d_{3} \Delta u_{3}=\frac{c m_{2} u_{3}}{c+u_{3}}-b_{2} u_{3}\end{cases}
$$

By Lemma 4.2, from the first and third equations in (4.7), we find that $u_{1}$ and $u_{3}$ are both constants:

$$
u_{1} \equiv \frac{1}{2}\left\{1-c+\sqrt{(1-c)^{2}+4 c\left(1-a_{1}\right)}\right\} \text { and } u_{3} \equiv \frac{m_{2}-b_{2}}{b_{2}} c .
$$

Substituting (4.8) into the second equation in (4.7), we find that $\left(u_{1}, c, u_{3}\right)=\tilde{\mathbf{u}}$. This verifies that the convergence holds for a subsequence of $\left(u_{1 i}, u_{2 i}, u_{3 i}\right)$. But the limit is a fixed point; thus the convergence holds for the whole sequence $\left(u_{1 i}, u_{2 i}, u_{3 i}\right)$.

Now we state our main result in this subsection.

THEOREM 4.4. Assume that $a_{1}<1$ and that assumptions in Theorem 4.3 hold.

(i) Let $\epsilon_{1}$ be an arbitrary positive constant. Then there exists $D_{2}=D_{2}\left(\epsilon_{1}, \Lambda, \Omega\right)$ such that (2.5) has no nonconstant positive solution provided that $\min \left\{d_{1}, d_{3}\right\} \geq \epsilon_{1}$ and $d_{2} \geq D_{2}$.

(ii) Let $\epsilon_{2}$ be an arbitrary positive constant. Then there exists $D_{1,3}=D_{1,3}\left(\epsilon_{2}, \Lambda, \Omega\right)$ such that (2.5) has no nonconstant positive solution provided that $d_{2} \geq \epsilon_{2}$ and $\min \left\{d_{1}, d_{3}\right\} \geq D_{1,3}$.

Proof. We first prove (i). By (i) of Theorem 4.2, for a fixed large constant $D_{1,3}$ depending only on $\Lambda$ and $\Omega$, there exists $D_{2}^{*}=D_{2}^{*}(\Lambda, \Omega)$ such that (2.1) has no positive nonconstant solution when $d_{1}, d_{3} \geq D_{1,3}$ and $d_{2} \geq D_{2}^{*}$. As a result, it suffices to consider the case $d_{1}, d_{3} \in\left[\epsilon_{1} / 2, D_{1,3}\right]$.

We make a decomposition: $u_{2}=w_{2}+\xi$ with $\int_{\Omega} w_{2}=0$ and $\xi \in \mathbf{R}^{+}$. We observe that finding the positive solution of (2.5) is equivalent to solving the following problem:

$$
\begin{cases}d_{1} \Delta u_{1}+u_{1}\left(1-u_{1}\right)-\frac{a_{1} u_{1}\left(w_{2}+\xi\right)}{u_{1}+w_{2}+\xi}=0 & \text { in } \Omega, \partial_{\nu} u_{1}=0 \text { on } \partial \Omega, \\ \Delta w_{2}+\rho\left\{\frac{m_{1} u_{1}\left(w_{2}+\xi\right)}{u_{1}+w_{2}+\xi}\right. & \text { in } \Omega, \partial_{\nu} w_{2}=0 \text { on } \partial \Omega, \\ \left.-b_{1}\left(w_{2}+\xi\right)-\frac{a_{2}\left(w_{2}+\xi\right) u_{3}}{w_{2}+\xi+u_{3}}\right\}=0 & \\ \int_{\Omega}\left\{\frac{m_{1} u_{1}\left(w_{2}+\xi\right)}{u_{1}+w_{2}+\xi}-b_{1}\left(w_{2}+\xi\right)\right. & \text { in } \Omega, \partial_{\nu} u_{3}=0 \text { on } \partial \Omega, \\ \left.-\frac{a_{2}\left(w_{2}+\xi\right) u_{3}}{w_{2}+\xi+u_{3}}\right\} \mathrm{d} x=0, & \text { in } \Omega,\end{cases}
$$

Copyright $@$ by SIAM. Unauthorized reproduction of this article is prohibited. 
where $\rho=d_{2}^{-1}$. Clearly, $\left(u_{1}, w_{2}, \xi, u_{3}\right)=\left(\tilde{u}_{1}, 0, \tilde{u}_{2}, \tilde{u}_{3}\right)$ is a solution of (4.9) for $\rho>0$.

To prove our theorem, by the finite covering argument, it is sufficient to prove that, for any fixed $\tilde{d}_{1}, \tilde{d}_{3} \in\left[\epsilon_{1} / 2, D_{1,3}\right]$, there exists $\delta_{0}>0$ such that if $\rho \in\left(0, \delta_{0}\right),\left(d_{1}, d_{3}\right) \in$ $\left(\tilde{d}_{1}-\delta_{0}, \tilde{d}_{1}+\delta_{0}\right) \times\left(\tilde{d}_{3}-\delta_{0}, \tilde{d}_{3}+\delta_{0}\right)$, then $\left(\tilde{u}_{1}, 0, \tilde{u}_{2}, \tilde{u}_{3}\right)$ is the unique solution of $(4.9)$. To this end, we define the following Banach spaces:

$W_{\nu}^{2,2}(\Omega)=\left\{g \in W^{2,2}(\Omega) \mid \partial_{\nu} g=0 \quad\right.$ on $\left.\partial \Omega\right\}, \quad L_{0}^{2}(\Omega)=\left\{g \in L^{2}(\Omega) \mid \int_{\Omega} g \mathrm{~d} x=0\right\}$,

and denote

$$
F\left(d_{1}, d_{3}, \rho, u_{1}, w_{2}, \xi, u_{3}\right)=\left(f_{1}, f_{2}, f_{3}, f_{4}\right)\left(d_{1}, d_{3}, \rho, u_{1}, w_{2}, \xi, u_{3}\right)
$$

with

$$
\begin{aligned}
f_{1}\left(d_{1}, d_{3}, \rho, u_{1}, w_{2}, \xi, u_{3}\right) & =d_{1} \Delta u_{1}+u_{1}\left(1-u_{1}\right)-\frac{a_{1} u_{1}\left(w_{2}+\xi\right)}{u_{1}+w_{2}+\xi}, \\
f_{2}\left(d_{1}, d_{3}, \rho, u_{1}, w_{2}, \xi, u_{3}\right) & =\Delta w_{2}+\rho\left\{\frac{m_{1} u_{1}\left(w_{2}+\xi\right)}{u_{1}+w_{2}+\xi}-b_{1}\left(w_{2}+\xi\right)\right. \\
- & \left.\frac{a_{2}\left(w_{2}+\xi\right) u_{3}}{w_{2}+\xi+u_{3}}\right\} \\
f_{3}\left(d_{1}, d_{3}, \rho, u_{1}, w_{2}, \xi, u_{3}\right) & =\int_{\Omega}\left\{\frac{m_{1} u_{1}\left(w_{2}+\xi\right)}{u_{1}+w_{2}+\xi}-b_{1}\left(w_{2}+\xi\right)-\frac{a_{2}\left(w_{2}+\xi\right) u_{3}}{w_{2}+\xi+u_{3}}\right\} \mathrm{d} x, \\
f_{4}\left(d_{1}, d_{3}, \rho, u_{1}, w_{2}, \xi, u_{3}\right) & =d_{3} \Delta u_{3}+\frac{m_{2}\left(w_{2}+\xi\right) u_{3}}{w_{2}+\xi+u_{3}}-b_{2} u_{3} .
\end{aligned}
$$

Then

$$
\begin{aligned}
F: \mathbf{R}^{+} & \times \mathbf{R}^{+} \times \mathbf{R}^{+} \times W_{\nu}^{2,2}(\Omega) \times\left(L_{0}^{2}(\Omega) \cap W_{\nu}^{2,2}(\Omega)\right) \\
& \times \mathbf{R}^{+} \times W_{\nu}^{2,2}(\Omega) \rightarrow L^{2}(\Omega) \times L_{0}^{2}(\Omega) \times \mathbf{R} \times L^{2}(\Omega)
\end{aligned}
$$

is a well-defined mapping. It is clear that the solutions of (4.9) satisfy $F\left(d_{1}, d_{3}, \rho, u_{1}\right.$, $\left.w_{2}, \xi, u_{3}\right)=0$. Moreover, (4.9) has a unique solution $\left(u_{1}, w_{2}, \xi, u_{3}\right)=\left(\tilde{u}_{1}, 0, \tilde{u}_{2}, \tilde{u}_{3}\right)$ when $\rho=0$ and $\left(d_{1}, d_{3}\right)=\left(\tilde{d}_{1}, \tilde{d}_{3}\right)$ from the proof of (i) of Lemma 4.3. Obviously, $F$ is a differentiable mapping, and its partial derivative with respect to the last four arguments is

$$
\begin{gathered}
\Psi \equiv D_{\left(u_{1}, w_{2}, \xi, u_{3}\right)} F\left(\tilde{d}_{1}, \tilde{d}_{3}, 0, \tilde{u}_{1}, 0, \tilde{u}_{2}, \tilde{u}_{3}\right), \\
\Psi: W_{\nu}^{2,2}(\Omega) \times\left(L_{0}^{2}(\Omega) \cap W_{\nu}^{2,2}(\Omega)\right) \times \mathbf{R} \times W_{\nu}^{2,2}(\Omega) \rightarrow L^{2}(\Omega) \times L_{0}^{2}(\Omega) \times \mathbf{R} \times L^{2}(\Omega)
\end{gathered}
$$

with

$$
\Psi\left(v_{1}, v_{2}, \tau, v_{3}\right)=\left(\begin{array}{c}
\tilde{d}_{1} \Delta v_{1}+a_{11} v_{1}+a_{12}\left(v_{2}+\tau\right) \\
\Delta v_{2} \\
\int_{\Omega}\left\{a_{21} v_{1}+a_{22}\left(v_{2}+\tau\right)+a_{23} v_{3}\right\} \mathrm{d} x \\
\tilde{d}_{3} \Delta v_{3}+a_{32}\left(v_{2}+\tau\right)+a_{33} v_{3}
\end{array}\right),
$$

where $a_{i j}$ are given in section 3 .

Copyright (c) by SIAM. Unauthorized reproduction of this article is prohibited. 
We claim that $\Psi$ is an isomorphism operator. Assume that $\Psi\left(v_{1}, v_{2}, \tau, v_{3}\right)=$ $(0,0,0,0)$; then $v_{2}=0$. Note that $a_{1}<1$ implies $a_{11}<0$. Then from the equation of $v_{1}$, it follows that $v_{1} \equiv-a_{12} \tau / a_{11}$. Similarly, $v_{3} \equiv-a_{32} \tau / a_{33}$ since $a_{33}<0$ and $\tau \in \mathbf{R}$. We substitute these results into the integral equations satisfied by $\left(v_{1}, v_{2}, \tau, v_{3}\right)$ and obtain that

$$
\left(-\frac{a_{12} a_{21}}{a_{11}}+a_{22}-\frac{a_{23} a_{32}}{a_{33}}\right) \tau=0 .
$$

This is equivalent to $\operatorname{det}\left\{\mathbf{G}_{\mathbf{u}}(\tilde{\mathbf{u}})\right\} \tau=0$, where

$$
\operatorname{det}\left\{\mathbf{G}_{\mathbf{u}}(\tilde{\mathbf{u}})\right\}=-\left(a_{12} a_{21} a_{33}+a_{11} a_{23} a_{32}-a_{11} a_{22} a_{33}\right)=-\frac{m_{1} m_{2} \tilde{u}_{1}^{2} \tilde{u}_{2}^{2} \tilde{u}_{3}}{\left(\tilde{u}_{1}+\tilde{u}_{2}\right)^{2}\left(\tilde{u}_{2}+\tilde{u}_{3}\right)^{2}}<0
$$

by some basic computations. Therefore $\tau=0$, which implies that $\left(v_{1}, v_{2}, \tau, v_{3}\right)=$ $(0,0,0,0)$ and $\Psi$ is injective. On the other hand, for a given $h_{2} \in L_{0}^{2}(\Omega)$, the problem

$$
-\Delta u_{2}=h_{2} \text { in } \Omega, \quad u \in L_{0}^{2}(\Omega) \cap W_{\nu}^{2,2}(\Omega)
$$

has a unique solution. By using $\operatorname{det}\left\{\mathbf{G}_{\mathbf{u}}(\tilde{\mathbf{u}})\right\}<0$ again, one can also check that $\Psi$ is also surjective. Consequently $\Psi$ is an isomorphism.

By the implicit function theorem, there exist positive constants $\rho_{0}$ and $\delta_{0}$ such that, for each $\rho \in\left[0, \rho_{0}\right]$ and $\left(d_{1}, d_{3}\right) \in\left(\tilde{d}_{1}-\delta_{0}, \tilde{d}_{1}+\delta_{0}\right) \times\left(\tilde{d}_{3}-\delta_{0}, \tilde{d}_{3}+\delta_{0}\right)$, $\left(\tilde{u}_{1}, 0, \tilde{u}_{2}, \tilde{u}_{3}\right)$ is the unique solution of $F\left(d_{1}, d_{3}, \rho, u_{1}, w_{2}, \xi, u_{3}\right)=0$ in $B_{\delta_{0}}\left(\tilde{u}_{1}\right.$, $\left.0, \tilde{u}_{2}, \tilde{u}_{3}\right)$, where $B_{\delta_{0}}\left(\tilde{u}_{1}, 0, \tilde{u}_{2}, \tilde{u}_{3}\right)$ is the ball in $W_{\nu}^{2,2}(\Omega) \times\left(L_{0}^{2}(\Omega) \cap W_{\nu}^{2,2}(\Omega)\right) \times$ $\mathbf{R} \times W_{\nu}^{2,2}(\Omega)$ centered at $\left(\tilde{u}_{1}, 0, \tilde{u}_{2}, \tilde{u}_{3}\right)$ with radius $\delta_{0}$. Taking smaller $\rho_{0}$ and $\delta_{0}$ if necessary, we can conclude (i) by use of Lemma 4.3(i).

In a similar manner, (ii) can be proved. In fact, we write $u_{i}=w_{i}+\xi_{i}$ with $\int_{\Omega} w_{i}=0$ and $\xi_{i} \in \mathbf{R}^{+}(i=1,3)$ and construct analogous operator

$$
F\left(d_{2}, \rho_{1}, \rho_{3}, w_{1}, \xi_{1}, u_{2}, w_{3}, \xi_{3}\right)=\left(f_{1}, f_{2}, f_{3}, f_{4}, f_{5}\right)\left(d_{2}, \rho_{1}, \rho_{3}, w_{1}, \xi_{1}, u_{2}, w_{3}, \xi_{3}\right)
$$

with

$f_{1}\left(d_{2}, \rho_{1}, \rho_{3}, w_{1}, \xi_{1}, u_{2}, w_{3}, \xi_{3}\right)=\Delta w_{1}$

$$
\begin{aligned}
& +\rho_{1}\left\{\left(w_{1}+\xi_{1}\right)\left(1-w_{1}-\xi_{1}\right)-\frac{a_{1}\left(w_{1}+\xi_{1}\right) u_{2}}{w_{1}+\xi_{1}+u_{2}}\right\}, \\
f_{2}\left(d_{2}, \rho_{1}, \rho_{3}, w_{1}, \xi_{1}, u_{2}, w_{3}, \xi_{3}\right)= & \int_{\Omega}\left\{\left(w_{1}+\xi_{1}\right)\left(1-w_{1}-\xi_{1}\right)-\frac{a_{1}\left(w_{1}+\xi_{1}\right) u_{2}}{w_{1}+\xi_{1}+u_{2}}\right\} \mathrm{d} x, \\
f_{3}\left(d_{2}, \rho_{1}, \rho_{3}, w_{1}, \xi_{1}, u_{2}, w_{3}, \xi_{3}\right)= & d_{2} \Delta u_{2}+\frac{m_{1}\left(w_{1}+\xi_{1}\right) u_{2}}{w_{1}+\xi_{1}+u_{2}}-b_{1} u_{2}-\frac{a_{2}\left(w_{3}+\xi_{3}\right) u_{2}}{w_{3}+\xi_{3}+u_{2}}, \\
f_{4}\left(d_{2}, \rho_{1}, \rho_{3}, w_{1}, \xi_{1}, u_{2}, w_{3}, \xi_{3}\right)= & \Delta w_{3}+\rho_{3}\left\{\frac{m_{2}\left(w_{3}+\xi_{3}\right) u_{2}}{w_{3}+\xi_{3}+u_{2}}-b_{2}\left(w_{3}+\xi_{3}\right)\right\}, \\
f_{5}\left(d_{2}, \rho_{1}, \rho_{3}, w_{1}, \xi_{1}, u_{2}, w_{3}, \xi_{3}\right)= & \int_{\Omega}\left\{\frac{m_{2}\left(w_{3}+\xi_{3}\right) u_{2}}{w_{3}+\xi_{3}+u_{2}}-b_{2}\left(w_{3}+\xi_{3}\right)\right\} \mathrm{d} x,
\end{aligned}
$$


where $\rho_{i}=d_{i}^{-1}(\mathrm{i}=1,3)$. For fixed $\tilde{d}_{2}>0$, we can verify that

$$
\begin{aligned}
& D_{\left(w_{1}, \xi_{1}, u_{2}, w_{3}, \xi_{3}\right)} F\left(\tilde{d}_{2}, 0,0,0, \tilde{u}_{1}, \tilde{u}_{2}, 0, \tilde{u}_{3}\right): \\
\left(L_{0}^{2}(\Omega) \cap W_{\nu}^{2,2}(\Omega)\right) & \times \mathbf{R} \times W_{\nu}^{2,2}(\Omega) \times\left(L_{0}^{2}(\Omega) \cap W_{\nu}^{2,2}(\Omega)\right) \\
& \times \mathbf{R} \rightarrow L_{0}^{2}(\Omega) \times \mathbf{R} \times L^{2}(\Omega) \times L_{0}^{2}(\Omega) \times \mathbf{R}
\end{aligned}
$$

is an isomorphism. As in the discussion of (i), by the implicit function theorem, Lemma 4.3(ii) and Theorem 4.2(ii), our result is obtained. The proof of Theorem 4.4 is complete.

5. Existence of nonconstant positive solutions of (2.5). This section is devoted to the existence of nonconstant positive solutions of (2.5) for certain values of diffusion coefficients $d_{1}$ and $d_{3}$, respectively, while the other parameters are fixed. Our results will show that, if the parameters are properly chosen, both the general stationary pattern and more interesting Turing pattern can arise as a result of diffusion.

For our purposes, we start with some preliminary results. First we study the linearization of $(2.5)$ at $\tilde{\mathbf{u}}$. We denote

$$
\mathbf{X}=\left\{\mathbf{u} \in\left[C^{2}(\bar{\Omega})\right]^{3} \mid \partial_{\nu} \mathbf{u}=0 \text { on } \partial \Omega\right\}
$$

and

$$
\begin{gathered}
\mathbf{X}^{+}=\left\{\mathbf{u} \in \mathbf{X} \mid u_{i}>0 \text { on } \bar{\Omega}, \quad i=1,2,3\right\}, \\
B(C)=\left\{\mathbf{u} \in \mathbf{X} \mid C^{-1}<u_{i}<C \text { on } \bar{\Omega}, \quad i=1,2,3\right\}, \quad C>0 .
\end{gathered}
$$

With the diffusion matrix $\mathcal{D}=\operatorname{diag}\left(d_{1}, d_{2}, d_{3}\right),(2.5)$ can be written as

$$
\begin{cases}-\mathcal{D} \Delta \mathbf{u}=\mathbf{G}(\mathbf{u}) & \text { in } \Omega, \\ \partial_{\nu} \mathbf{u}=0 & \text { on } \partial \Omega,\end{cases}
$$

and $\mathbf{u}$ is a positive solution to (5.1) if and only if

$$
\mathbf{F}(\mathbf{u}) \equiv \mathbf{u}-(\mathbf{I}-\Delta)^{-1}\left\{\mathcal{D}^{-1} \mathbf{G}(\mathbf{u})+\mathbf{u}\right\}=0 \text { for } \mathbf{u} \in \mathbf{X}^{+},
$$

where $(\mathbf{I}-\Delta)^{-1}$ is the inverse of $\mathbf{I}-\Delta$ in $\mathbf{X}$. As $\mathbf{F}(\cdot)$ is a compact perturbation of the identity operator, for any $B=B(C)$, the Leray-Schauder degree $\operatorname{deg}(\mathbf{F}(\cdot), 0, B)$ is well defined if $\mathbf{F}(\mathbf{u}) \neq 0$ on $\partial B$.

We also note that

$$
D_{\mathbf{u}} \mathbf{F}(\tilde{\mathbf{u}})=\mathbf{I}-(\mathbf{I}-\Delta)^{-1}\left\{\mathcal{D}^{-1} \mathbf{G}_{\mathbf{u}}(\tilde{\mathbf{u}})+\mathbf{I}\right\},
$$

and recall that if $D_{\mathbf{u}} \mathbf{F}(\tilde{\mathbf{u}})$ is invertible, the index of $\mathbf{F}$ at $\tilde{\mathbf{u}}$ is defined as $\operatorname{index}(\mathbf{F}(\cdot), \tilde{\mathbf{u}})=$ $(-1)^{\gamma}$, where $\gamma$ is the multiplicity of negative eigenvalues of $D_{\mathbf{u}} \mathbf{F}(\tilde{\mathbf{u}})[25$, Theorem 2.8.1].

For the sake of convenience, we denote

$$
H\left(d_{1}, d_{2}, d_{3} ; \mu\right) \equiv \operatorname{det}\left\{\mu \mathbf{I}-\mathcal{D}^{-1} \mathbf{G}_{\mathbf{u}}(\tilde{\mathbf{u}})\right\}=\frac{1}{d_{1} d_{2} d_{3}} \operatorname{det}\left\{\mu \mathcal{D}-\mathbf{G}_{\mathbf{u}}(\tilde{\mathbf{u}})\right\},
$$

By arguments similar to those in [28], it can be shown that the following proposition holds. 
Proposition 5.1. Suppose that, for all $n \geq 0$, the matrix $\mu_{n} \mathbf{I}-\mathcal{D}^{-1} \mathbf{G}_{\mathbf{u}}(\tilde{\mathbf{u}})$ is nonsingular. Then

$$
\operatorname{index}(\mathbf{F}(\cdot), \tilde{\mathbf{u}})=(-1)^{\gamma}, \quad \text { where } \gamma=\sum_{n \geq 0, H\left(d_{1}, d_{2}, d_{3} ; \mu_{n}\right)<0} \operatorname{dim} E\left(\mu_{n}\right) .
$$

To compute index $(\mathbf{F}(\cdot), \tilde{\mathbf{u}})$, we have to consider the sign of $H\left(d_{1}, d_{2}, d_{3} ; \mu\right)$. Direct calculation gives

$$
\begin{aligned}
\operatorname{det}\left\{\mu \mathcal{D}-\mathbf{G}_{\mathbf{u}}(\tilde{\mathbf{u}})\right\}= & A_{3}\left(d_{1}, d_{3}\right) \mu^{3}+A_{2}\left(d_{1}, d_{3}\right) \mu^{2}+A_{1}\left(d_{1}, d_{3}\right) \mu \\
& -\operatorname{det}\left\{\mathbf{G}_{\mathbf{u}}(\tilde{\mathbf{u}})\right\} \equiv \mathcal{A}\left(d_{1}, d_{3} ; \mu\right),
\end{aligned}
$$

with

$$
\left\{\begin{array}{l}
A_{3}\left(d_{1}, d_{3}\right)=d_{1} d_{2} d_{3}, \quad A_{2}\left(d_{1}, d_{3}\right)=-\left\{a_{33} d_{1} d_{2}+\left(a_{11} d_{2}+a_{22} d_{1}\right) d_{3}\right\}, \\
A_{1}\left(d_{1}, d_{3}\right)=a_{11} a_{33} d_{2}+\left(a_{22} a_{33}-a_{23} a_{32}\right) d_{1}+\left(a_{11} a_{22}-a_{12} a_{21}\right) d_{3},
\end{array}\right.
$$

where $a_{i j}$ are defined in section 3 .

We first consider the dependence of $\mathcal{A}$ on $d_{1}$. Let $\tilde{\mu}_{i}\left(d_{1} ; d_{2}, d_{3}\right), i=1,2,3$, be the three roots of $\mathcal{A}\left(d_{1}, d_{3} ; \mu\right)=0$ satisfying $\operatorname{Re}\left\{\tilde{\mu}_{1}\left(d_{1} ; d_{2}, d_{3}\right)\right\} \leq \operatorname{Re}\left\{\tilde{\mu}_{2}\left(d_{1} ; d_{2}, d_{3}\right)\right\} \leq$ $\operatorname{Re}\left\{\tilde{\mu}_{3}\left(d_{1} ; d_{2}, d_{3}\right)\right\}$. Since $\operatorname{det}\left\{\mathbf{G}_{\mathbf{u}}(\tilde{\mathbf{u}})\right\}<0$ and $A_{3}\left(d_{1}, d_{3}\right)>0$, one of $\tilde{\mu}_{i}\left(d_{1} ; d_{2}, d_{3}\right)$ is real and negative, and the product of the other two is positive.

In addition, we have

$$
\lim _{d_{1} \rightarrow \infty} \mathcal{A}\left(d_{1}, d_{3} ; \mu\right) / d_{1}=\mu\left[d_{2} d_{3} \mu^{2}-\left(a_{33} d_{2}+a_{22} d_{3}\right) \mu+a_{22} a_{33}-a_{23} a_{32}\right] .
$$

Note that $a_{22} a_{33}-a_{23} a_{32}>0$. If $a_{22}>0$ or the reverse inequality of (3.5),

$$
m_{1}<\left(b_{1}+a_{2} \frac{m_{2}-b_{2}}{m_{2}}\right)^{2} /\left(b_{1}+a_{2}\left(\frac{m_{2}-b_{2}}{m_{2}}\right)^{2}\right)
$$

holds, and the parameters $d_{2}$ and $d_{3}$ satisfy

$$
a_{33} d_{2}+a_{22} d_{3}>0, \quad \Delta_{1} \equiv\left(a_{33} d_{2}+a_{22} d_{3}\right)^{2}-4 d_{2} d_{3}\left(a_{22} a_{33}-a_{23} a_{32}\right)>0,
$$

we can establish the following proposition.

Proposition 5.2. Assume that (5.4) holds and that $d_{2}$ and $d_{3}$ satisfy (5.5). Then there exists a positive constant $D_{1}^{*}$ such that when $d_{1} \geq D_{1}^{*}$, the three roots $\tilde{\mu}_{i}\left(d_{1} ; d_{2}\right.$, $\left.d_{3}\right), i=1,2,3$, of $\mathcal{A}\left(d_{1}, d_{3} ; \mu\right)=0$ are all real and satisfy

$$
\left\{\begin{array}{l}
\lim _{d_{1} \rightarrow \infty} \tilde{\mu}_{1}\left(d_{1} ; d_{2}, d_{3}\right)=0 \\
\lim _{d_{1} \rightarrow \infty} \tilde{\mu}_{2}\left(d_{1} ; d_{2}, d_{3}\right)=\frac{1}{2 d_{2} d_{3}}\left\{a_{33} d_{2}+a_{22} d_{3}-\sqrt{\Delta_{1}}\right\} \equiv \mu_{2}^{*}\left(d_{2}, d_{3}\right)>0 \\
\lim _{d_{1} \rightarrow \infty} \tilde{\mu}_{3}\left(d_{1} ; d_{2}, d_{3}\right)=\frac{1}{2 d_{2} d_{3}}\left\{a_{33} d_{2}+a_{22} d_{3}+\sqrt{\Delta_{1}}\right\} \equiv \mu_{3}^{*}\left(d_{2}, d_{3}\right)>0 .
\end{array}\right.
$$

Moreover, when $d_{1} \geq D_{1}^{*}$,

$$
\left\{\begin{array}{l}
-\infty<\tilde{\mu}_{1}\left(d_{1} ; d_{2}, d_{3}\right)<0<\tilde{\mu}_{2}\left(d_{1} ; d_{2}, d_{3}\right)<\tilde{\mu}_{3}\left(d_{1} ; d_{2}, d_{3}\right) \\
\mathcal{A}\left(d_{1}, d_{3} ; \mu\right)<0 \text { if } \mu \in\left(-\infty, \tilde{\mu}_{1}\left(d_{1} ; d_{2}, d_{3}\right)\right) \cup\left(\tilde{\mu}_{2}\left(d_{1} ; d_{2}, d_{3}\right), \tilde{\mu}_{3}\left(d_{1} ; d_{2}, d_{3}\right)\right) \\
\mathcal{A}\left(d_{1}, d_{3} ; \mu\right)>0 \text { if } \mu \in\left(\tilde{\mu}_{1}\left(d_{1} ; d_{2}, d_{3}\right), \tilde{\mu}_{2}\left(d_{1} ; d_{2}, d_{3}\right)\right) \cup\left(\tilde{\mu}_{3}\left(d_{1} ; d_{2}, d_{3}\right), \infty\right)
\end{array}\right.
$$

Copyright $@$ ㅇ by SIAM. Unauthorized reproduction of this article is prohibited. 
Similarly, we consider $d_{3}$ as the parameter, and $d_{1}$ and $d_{2}$ satisfy

$$
a_{11} d_{2}+a_{22} d_{1}>0, \quad \Delta_{2} \equiv\left(a_{11} d_{2}+a_{22} d_{1}\right)^{2}-4 d_{1} d_{2}\left(a_{11} a_{22}-a_{12} a_{21}\right)>0 ;
$$

then we have the following proposition.

Proposition 5.3. Assume that (5.4) holds and that $d_{1}$ and $d_{2}$ satisfy (5.8). Then there exists a positive constant $D_{3}^{*}$ such that when $d_{3} \geq D_{3}^{*}$, the three roots $\tilde{\mu}_{1}\left(d_{3} ; d_{1}\right.$, $\left.d_{2}\right), i=1,2,3$, of $\mathcal{A}\left(d_{1}, d_{3} ; \mu\right)=0$ are all real and satisfy

$$
\left\{\begin{array}{l}
\lim _{d_{3} \rightarrow \infty} \tilde{\mu}_{1}\left(d_{3} ; d_{1}, d_{2}\right) \leq 0 \\
\lim _{d_{3} \rightarrow \infty} \tilde{\mu}_{2}\left(d_{3} ; d_{1}, d_{2}\right)=\frac{1}{2 d_{1} d_{2}}\left\{a_{11} d_{2}+a_{22} d_{1}-\sqrt{\Delta_{2}}\right\} \equiv \mu_{2}^{*}\left(d_{1}, d_{2}\right) \geq 0, \\
\lim _{d_{3} \rightarrow \infty} \tilde{\mu}_{3}\left(d_{3} ; d_{1}, d_{2}\right)=\frac{1}{2 d_{1} d_{2}}\left\{a_{11} d_{2}+a_{22} d_{1}+\sqrt{\Delta_{2}}\right\} \equiv \mu_{3}^{*}\left(d_{1}, d_{2}\right)>0 .
\end{array}\right.
$$

Moreover, when $d_{3} \geq D_{3}^{*}$,

$$
\left\{\begin{array}{l}
-\infty<\tilde{\mu}_{1}\left(d_{3} ; d_{1}, d_{2}\right)<0<\tilde{\mu}_{2}\left(d_{3} ; d_{1}, d_{2}\right)<\tilde{\mu}_{3}\left(d_{3} ; d_{1}, d_{2}\right), \\
\mathcal{A}\left(d_{1}, d_{3} ; \mu\right)<0 \text { if } \mu \in\left(-\infty, \tilde{\mu}_{1}\left(d_{3} ; d_{1}, d_{2}\right)\right) \cup\left(\tilde{\mu}_{2}\left(d_{3} ; d_{1}, d_{2}\right), \tilde{\mu}_{3}\left(d_{3} ; d_{1}, d_{2}\right)\right), \\
\mathcal{A}\left(d_{1}, d_{3} ; \mu\right)>0 \text { if } \mu \in\left(\tilde{\mu}_{1}\left(d_{3} ; d_{1}, d_{2}\right), \tilde{\mu}_{2}\left(d_{3} ; d_{1}, d_{2}\right)\right) \cup\left(\tilde{\mu}_{3}\left(d_{3} ; d_{1}, d_{2}\right), \infty\right) .
\end{array}\right.
$$

Remark 5.1. Simple computations show that $\mu_{2}^{*}\left(d_{1}, d_{2}\right)=0$ if and only if $a_{11} a_{22}-$ $a_{12} a_{21} \leq 0$.

In virtue of Theorems 4.3 and 4.2 and Propositions 5.1 and 5.2, the first result of the existence of nonconstant positive solutions of (2.5) can be stated as follows.

TheOREM 5.1. Assume that $a_{1}<1,(5.4),(5.5)$, and assumptions in Theorem 4.3 hold. If $\mu_{2}^{*}\left(d_{2}, d_{3}\right) \in\left(\mu_{i}, \mu_{i+1}\right)$ and $\mu_{3}^{*}\left(d_{2}, d_{3}\right) \in\left(\mu_{j}, \mu_{j+1}\right)$ for some $j>i \geq 0$, where $\mu_{2}^{*}\left(d_{2}, d_{3}\right), \mu_{3}^{*}\left(d_{2}, d_{3}\right)$ are defined in Proposition 5.2, and the sum $\sum_{n=i+1}^{j} \operatorname{dim} E\left(\mu_{n}\right)$ is odd, then there exists a positive constant $\tilde{D}_{1}$ such that, if $d_{1} \geq \tilde{D}_{1},(2.5)$ admits at least one nonconstant positive solution.

Proof. By Proposition 5.2 and our assumptions, there exists a positive constant $\tilde{D}_{1}$, such that when $d_{1} \geq \tilde{D}_{1},(5.7)$ holds and

$$
\mu_{i}<\tilde{\mu}_{2}\left(d_{1} ; d_{2}, d_{3}\right)<\mu_{i+1}, \quad \mu_{j}<\tilde{\mu}_{3}\left(d_{1} ; d_{2}, d_{3}\right)<\mu_{j+1} .
$$

According to Theorem 4.2 , for $\hat{d}_{1}$ and $\hat{d}_{3}$ satisfying $\mu_{1} \hat{d}_{1}>1, \mu_{1} \hat{d}_{3}>m_{2}-b_{2}$, there exists a large $\hat{d}_{2}$ such that (2.5) has no constant positive solutions when $d_{1} \geq \hat{d}_{1}$, $\mu_{1} d_{2} \geq \hat{d}_{2}$, and $d_{3} \geq \hat{d}_{3}$. In addition, since $\operatorname{det}\left\{\mathbf{G}_{\mathbf{u}}(\tilde{\mathbf{u}})\right\}<0$ and $\lim _{n \rightarrow \infty} \mu_{n}=\infty$, from (5.3), we can further choose $\hat{d}_{1}, \hat{d}_{2}$, and $\hat{d}_{3}$ to be so large that

$$
H\left(\hat{d}_{1}, \hat{d}_{2}, \hat{d}_{3} ; \mu_{n}\right)>0 \quad \text { for all } n \geq 0 .
$$

Now we show that for any $d_{1} \geq \tilde{D}_{1},(2.5)$ has at least one nonconstant positive solution. The proof, which is accomplished by a contradiction argument, is based on the homotopy invariance of the topological degree. Suppose on the contrary that the assertion is not true for some $d_{1}=\tilde{d}_{1} \geq \tilde{D}_{1}$.

We fix $d_{1}=\tilde{d}_{1}$. Let $d_{i}(t)=t d_{i}+(1-t) \hat{d}_{i}, i=1,2,3$, and define $\mathcal{D}(t)=$ $\operatorname{diag}\left(d_{1}(t), d_{2}(t), d_{3}(t)\right)$. Now we consider the problem

$$
\begin{cases}-\mathcal{D}(t) \Delta \mathbf{u}=\mathbf{G}(\mathbf{u}) & \text { in } \Omega, \\ \partial_{\nu} \mathbf{u}=0 & \text { on } \partial \Omega .\end{cases}
$$

Copyright $@$ by SIAM. Unauthorized reproduction of this article is prohibited. 
Then $\mathbf{u}$ is a positive solution of (2.5) if and only if it is a positive solution of (5.11) for $t=1$. It is obvious that $\tilde{\mathbf{u}}$ is the unique constant positive solution of (5.11) for any $0 \leq t \leq 1$. For any $0 \leq t \leq 1, \mathbf{u}$ is a positive solution of (5.11) if and only if

$$
\mathbf{F}(t ; \mathbf{u}) \equiv \mathbf{u}-(\mathbf{I}-\Delta)^{-1}\left\{\mathcal{D}^{-1}(t) \mathbf{G}(\mathbf{u})+\mathbf{u}\right\}=0 \text { for } \mathbf{u} \in \mathbf{X}^{+}
$$

Clearly, $\mathbf{F}(1 ; \mathbf{u})=\mathbf{F}(\mathbf{u})$. Theorem 4.2 shows that the only positive solution of $\mathbf{F}(0 ; \mathbf{u})=0$ is $\tilde{\mathbf{u}}$. From direct calculation,

$$
D_{\mathbf{u}} \mathbf{F}(t ; \tilde{\mathbf{u}})=\mathbf{I}-(\mathbf{I}-\Delta)^{-1}\left\{\mathcal{D}^{-1}(t) \mathbf{G}_{\mathbf{u}}(\tilde{\mathbf{u}})+\mathbf{I}\right\} .
$$

In particular,

$$
\begin{aligned}
& D_{\mathbf{u}} \mathbf{F}(0 ; \tilde{\mathbf{u}})=\mathbf{I}-(\mathbf{I}-\Delta)^{-1}\left\{\hat{\mathcal{D}}^{-1} \mathbf{G}_{\mathbf{u}}(\tilde{\mathbf{u}})+\mathbf{I}\right\}, \\
& D_{\mathbf{u}} \mathbf{F}(1 ; \tilde{\mathbf{u}})=\mathbf{I}-(\mathbf{I}-\Delta)^{-1}\left\{\mathcal{D}^{-1} \mathbf{G}_{\mathbf{u}}(\tilde{\mathbf{u}})+\mathbf{I}\right\}=D_{\mathbf{u}} \mathbf{F}(\tilde{\mathbf{u}}),
\end{aligned}
$$

where $\hat{\mathcal{D}}=\operatorname{diag}\left(\hat{d}_{1}, \hat{d}_{2}, \hat{d}_{3}\right)$. From (5.2) and (5.3) we see that

$$
H\left(d_{1}, d_{2}, d_{3} ; \mu\right)=\frac{1}{d_{1} d_{2} d_{3}} \mathcal{A}\left(d_{1}, d_{3} ; \mu\right) .
$$

In view of (5.7) and (5.9), it follows from (5.12) that

$$
\left\{\begin{array}{l}
H\left(d_{1}, d_{2}, d_{3} ; \mu_{0}\right)=H(0)>0 \\
H\left(d_{1}, d_{2}, d_{3} ; \mu_{n}\right)<0, \quad i+1 \leq n \leq j \\
H\left(d_{1}, d_{2}, d_{3} ; \mu_{n}\right)>0, \quad 1 \leq n \leq i \text { and } n \geq j+1
\end{array}\right.
$$

Therefore, zero is not an eigenvalue of the matrix $\mu_{i} \mathbf{I}-\mathcal{D}^{-1} \mathbf{G}_{\mathbf{u}}(\tilde{\mathbf{u}})$ for all $n \geq 0$, and

$$
\sum_{n \geq 0, H\left(d_{1}, d_{2}, d_{3} ; \mu_{n}\right)<0} \operatorname{dim} E\left(\mu_{n}\right)=\sum_{n=i+1}^{j} \operatorname{dim} E\left(\mu_{n}\right)=\text { an odd number. }
$$

Then Proposition 5.1 shows that

$$
\operatorname{index}(\mathbf{F}(1 ; \cdot), \tilde{\mathbf{u}})=(-1)^{\gamma}=-1
$$

On the other hand, by (5.10) and Proposition 5.1 again, we obtain that

$$
\operatorname{index}(\mathbf{F}(0 ; \cdot), \tilde{\mathbf{u}})=(-1)^{0}=1 .
$$

In view of $\tilde{d}_{1}>\tilde{D}_{1}$, by Theorem 4.3 , there exists a positive constant $C=C\left(\tilde{D}_{1}, d_{2}\right.$, $\left.d_{3}, \hat{d}_{1}, \hat{d}_{2}, \hat{d}_{3}, \Lambda\right)$ such that, for all $0 \leq t \leq 1$, the positive solutions of (5.11) satisfy $1 / C<u_{1}, u_{2}, u_{3}<C$. Therefore, $\mathbf{F}(t ; \mathbf{u}) \neq 0$ on $\partial B(C)$ for all $0 \leq t \leq 1$. By the homotopy invariance of the topological degree,

$$
\operatorname{deg}(\mathbf{F}(1 ; \cdot), 0, B(C))=\operatorname{deg}(\mathbf{F}(0 ; \cdot), 0, B(C)) .
$$

Moreover, under our assumptions, the only positive solution of both $\mathbf{F}(1 ; \mathbf{u})=0$ and $\mathbf{F}(0 ; \mathbf{u})=0$ in $B(C)$ is $\tilde{\mathbf{u}}$, and hence, by (5.13) and (5.14),

$$
\operatorname{deg}(\mathbf{F}(0 ; \cdot), 0, B(C))=\operatorname{index}(\mathbf{F}(0 ; \cdot), \tilde{\mathbf{u}})=1
$$

and

$$
\operatorname{deg}(\mathbf{F}(1 ; \cdot), 0, B(C))=\operatorname{index}(\mathbf{F}(1 ; \cdot), \tilde{\mathbf{u}})=-1 .
$$

This contradicts (5.15), and the proof is complete. 
Remark 5.2. When $d_{3}$ is fixed, we note that $\lim _{d_{2} \rightarrow 0} \mu_{2}^{*}\left(d_{2}, d_{3}\right)=\left(a_{22} a_{33}-\right.$ $\left.a_{23} a_{32}\right) /\left(a_{22} d_{3}\right), \lim _{d_{2} \rightarrow 0} \mu_{3}^{*}\left(d_{2}, d_{3}\right)=\infty$, and (5.5) is automatically fulfilled for small $d_{2}$. Therefore, if for all $i=0,1,2, \ldots, \mu_{i}$ are simple and $\left(a_{22} a_{33}-a_{23} a_{32}\right) /\left(a_{22} d_{3}\right) \neq$ $\mu_{i}$, by Theorem 5.1, when $a_{1}<1$ and the assertion of Theorem 4.3 hold, there exist two sequences of intervals $\left\{\left(\theta_{n}^{1}, \theta_{n}^{2}\right)\right\}_{n=1}^{\infty}$ and $\left\{\left(\Theta_{n}^{1}, \Theta_{n}^{2}\right)\right\}_{n=1}^{\infty}$ satisfying $\theta_{n+1}^{2}<$ $\theta_{n}^{1}, \Theta_{n}^{2}<\Theta_{n+1}^{1}$, and $\theta_{n}^{1}, \theta_{n}^{2} \rightarrow 0^{+}$while $\Theta_{n}^{1}, \Theta_{n}^{2} \rightarrow \infty$ as $n \rightarrow \infty$ such that (2.5) admits at least one nonconstant positive solution for all $d_{1} \in\left(\Theta_{n}^{1}, \Theta_{n}^{2}\right)$ and $d_{2} \in$ $\left(\theta_{n}^{1}, \theta_{n}^{2}\right), n=1,2,3, \ldots$ Recall that Theorem 4.3 holds when condition (i) or (ii) in Lemma 4.1 holds. When (i) (same as (3.8)) holds, (5.4) is not satisfied. But when (ii) in Lemma 4.1 holds, conditions in Theorem 5.1 can be satisfied.

Similarly, let us consider the case of large $d_{3}$. By Proposition 5.3 and Remark 5.1, we have the following theorem.

Theorem 5.2. Assume that $a_{1}<1,(5.4),(5.8)$, and Theorem 4.3 hold.

(i) If $a_{11} a_{22}-a_{12} a_{21}>0$, then $\mu_{2}^{*}\left(d_{1}, d_{2}\right) \in\left(\mu_{i}, \mu_{i+1}\right), \mu_{3}^{*}\left(d_{1}, d_{2}\right) \in\left(\mu_{j}, \mu_{j+1}\right)$ for some $j>i \geq 0$, and $\sum_{n=i+1}^{j} \operatorname{dim} E\left(\mu_{n}\right)$ is odd.

(ii) If $a_{11} a_{22}-a_{12} a_{21} \leq 0, \mu_{3}^{*}\left(d_{1}, d_{2}\right) \in\left(\mu_{j}, \mu_{j+1}\right)$ for some $j>0$, and $\sum_{n=1}^{j} \operatorname{dim}$ $E\left(\mu_{n}\right)$ is odd, where $\mu_{2}^{*}\left(d_{1}, d_{2}\right), \mu_{3}^{*}\left(d_{1}, d_{2}\right)$ are defined in Proposition 5.3, then there exists a positive constant $\tilde{D}_{3}$ such that, if $d_{3} \geq \tilde{D}_{3}$, (2.5) admits at least one nonconstant positive solution.

Remark 5.3. By Proposition 5.3, regardless of the sign of $a_{11} a_{22}-a_{12} a_{21}$, we have a conclusion similar to that in Remark 5.2. In addition, we mention that the sign of $a_{11} a_{22}-a_{12} a_{21}$ is indefinite when $a_{1}<1, a_{22}>0, \tilde{\mathbf{u}}$ exists, and the assertion of Theorem 4.3 holds (note that, if $\sqrt{a_{2}+m_{2}}<\sqrt{m_{1}-b_{1}}+\sqrt{b_{2}}$, then Theorem 4.3 is true by Lemma 4.1). The detailed analysis on this claim is left to the appendix.

Remark 5.4. Fix $d_{1}$ and $d_{2}$; by Remark 3.1, if $m_{1}=\left(b_{1}+a_{2}\left(m_{2}-b_{2}\right) / m_{2}\right)^{2} /\left(b_{1}+\right.$ $\left.a_{2}\left(m_{2}-b_{2}\right)^{2} / m_{2}^{2}\right)-o\left(b_{1}\right)$, and $b_{1} \rightarrow 0, a_{2}, b_{2}, m_{2}$ are properly chosen and either $a_{1} \rightarrow 1 / 2$ or $a_{1} \rightarrow 1$, and $d_{3}$ is sufficiently large, Turing instability actually happens. Furthermore, combined with the analysis of the appendix, Proposition A.2(i) also holds for such chosen parameters. With proper choices of $d_{1}$ and $d_{2}$, we can find certain parameter ranges guaranteeing the existence of both Turing instability and the nonconstant positive solution to (2.5) by Theorem 5.2(i). As a consequence, Turing patterns exist for these parameter ranges.

6. Conclusions. In this paper, we analyze a reaction-diffusion food chain model with ratio-dependent functional response. We are mainly concerned with the coexistence of the three species and focus on the case of a weak predation rate for the pest species (i.e., $a_{1}<1$ ). In particular, the existence and nonexistence of nonconstant positive steady states have been established. The existence results provide a theoretical support for pattern formation caused by diffusion.

We summarize our investigation here and hope to reveal some interesting phenomena of pattern formation in population ecology. We always assume the existence of a constant coexistence. The main results of sections 3 and 4 show that this constant coexistence steady state $\tilde{\mathbf{u}}$ is the only steady state if (a) both of the predation rates $a_{1}$ and $a_{2}$ are small; (b) $a_{1}$ is small while $a_{2}$ is suitably chosen, and either the pest or the other two species diffuse quickly (Theorem 4.4). In the former case, we are also able to find a more restrictive parameter range so that $\tilde{\mathbf{u}}$ is globally asymptotically stable (Theorem 3.3). This can also be seen from a bifurcation point of view. Here if we assume that $a_{1}$ is small, then stronger stability results of $\tilde{\mathbf{u}}$ can be proved when $a_{2}$ is smaller. When $a_{2}$ is close to zero, then $\tilde{\mathbf{u}}$ is globally asymptotically stable; when $a_{2}$ 
increases, $\tilde{\mathbf{u}}$ is still locally asymptotically stable but may not be globally asymptotically stable, and it is still the only steady state; and when $a_{2}$ further increases, $\tilde{\mathbf{u}}$ becomes unstable for both the ODE and the reaction-diffusion system, and nonconstant patterns exist in this case. In the latter case, the diffusion of the first or third species must be large enough (see Theorems 5.1 and 5.2). Thus for small $a_{1}$ and suitable $a_{2}$, the quick migration of the plant or top predator enhances the formation of spatial pattern for the system. In contrast, the quick migration of the pest or both the plant and top predators tends to prevent the system from generating pattern. It is well known that fast diffusion of all species in a biological system will not lead to spatial inhomogeneous patterns; see [6]. Our result shows the importance of the diffusion rate of the middle species in a food chain. The large diffusion rate of the pest (middle species) alone can lead to the nonexistence of spatial patterns, but if the pest diffusion rate is not large, then all other diffusion rates must be large to prevent the occurrence of patterns. On the other hand, a large diffusion rate of the top species or bottom species will help the generation of patterns. This demonstrates that, in an ecological model, different diffusions may play essentially different roles in developing spatial patterns. In addition, taking into account the close relationship between the time-dependent solutions to a reaction-diffusion system and the corresponding steady state solutions, to a great extent, the dynamical behaviors of (2.4) will be determined by the diffusions of the three species.

These conclusions can also be compared with those in [28] and [36]. In the absence of $u_{3},(2.4)$ becomes the prey-predator model studied by Pang and Wang in [28]. The results of the existence and nonexistence of nonconstant positive solutions there indicate that large $d_{2}$ contributes to the evolution of heterogeneousness for the dynamics, while large $d_{1}$ tends to increase the possibility of spatial uniform distribution. Therefore, combined with the our conclusions for (2.5), this suggests that the structure of solutions to the model in [28] will be significantly different due to the emergence of the top predator, which in turn leads to the qualitative change of the biological mechanism of the system. Such a phenomenon was also discussed by Lou, Martinez, and $\mathrm{Ni}$ for the classical Lotka-Volterra competition model in [20].

In [36], Wang investigated a three-species prey-predator model. In that model, the interaction between the lower and middle species is described by Holling II-type functional response (prey-dependent), while the functional response between the middle and top species is ratio-dependent (predator-dependent). It was proved that Turing pattern may appear if both $d_{1}$ and $d_{3}$ are large, but will not if $d_{2}$ is large. Therefore the results of the present paper and [36] show that the formation of Turing pattern in the biological models with the same degree of complexity depends on the choices of functional responses. In other words, the feeding strategy of predators may be one of the determining factors in producing Turing pattern. In a very recent work [2], Alonso, Bartumeus, and Catalan performed some numerical calculations indicating that predator-dependent models are sometimes capable of generating Turing pattern, while similar prey-dependent models are not. Hence our theoretical analysis for the food chain model rigorously confirms the outcome of computer simulation in $[2]$.

Finally we point out that some of our mathematical techniques in sections 4 and 5 can be applied to deal with the prey-predator model proposed by Pang and Wang in [28] and derive some new a priori estimates for positive steady state solutions and nonexistence results for nonconstant positive steady state solutions.

Appendix A. In section 6, to prove the existence of nonconstant positive solutions to $(2.5)$, we have made some hypotheses, namely, $a_{1}<1, a_{22}>0$, $\tilde{\mathbf{u}}$ exists, and The- 
orem 4.3 holds (in particular, $\sqrt{a_{2}+m_{2}}<\sqrt{m_{1}-b_{1}}+\sqrt{b_{2}}$ means that Theorem 4.3 is true). We list these conditions as follows:

$$
\left\{\begin{array}{l}
a_{1}<1, \quad m_{2}>b_{2}, \quad A>1 \Longleftrightarrow a_{2}\left(m_{2}-b_{2}\right) / m_{2}+b_{1}<m_{1}, \\
\sqrt{a_{2}+m_{2}}<\sqrt{m_{1}-b_{1}}+\sqrt{b_{2}} \Longleftrightarrow\left(\sqrt{a_{2}+m_{2}}-\sqrt{b_{2}}\right)^{2}+b_{1}<m_{1}, \\
a_{22}>0 \Longleftrightarrow m_{1}<\left(b_{1}+a_{2}\left(m_{2}-b_{2}\right) / m_{2}\right)^{2} /\left(b_{1}+a_{2}\left(m_{2}-b_{2}\right)^{2} / m_{2}^{2}\right)
\end{array}\right.
$$

In the following, we will verify the claim made in Remark 5.3, which says that $a_{11} a_{22}-a_{12} a_{21}$ is indefinite when (A.1) holds. First of all, by the definitions of $a_{11}, a_{22}, a_{12}, a_{21}$, the direct computations yield the following proposition.

Proposition A.1. Define

$$
\begin{aligned}
Q \equiv & -\left(1-a_{1}\right) a_{2} b_{2}\left(m_{2}-b_{2}\right) A^{3}+\left(1-a_{1}\right) m_{1} m_{2}^{2} A^{2} \\
& +\left[\left(2 a_{1}-1\right) m_{1} m_{2}^{2}-a_{1} a_{2} b_{2}\left(m_{2}-b_{2}\right)\right] A-a_{1} m_{1} m_{2}^{2} ;
\end{aligned}
$$

then $a_{11} a_{22}-a_{12} a_{21}>0 \Longleftrightarrow Q>0$. Moreover, we note that

(i) as $a_{1} \rightarrow 0, Q \rightarrow\left(m_{1} m_{2}^{2}(A-1)-a_{2} b_{2}\left(m_{2}-b_{2}\right) A^{2}\right) A$;

(ii) as $a_{1} \rightarrow 1 / 2, Q \rightarrow-\frac{1}{2} a_{2} b_{2}\left(m_{2}-b_{2}\right) A^{3}-\frac{1}{2} a_{2} b_{2}\left(m_{2}-b_{2}\right) A+\frac{1}{2} m_{1} m_{2}^{2}(A+$ 1) $(A-1)$

(iii) as $a_{1} \rightarrow 1, Q \rightarrow m_{1} m_{2}^{2}(A-1)-a_{2} b_{2}\left(m_{2}-b_{2}\right) A$.

Proposition A.2. The following results hold:

(i) If we take $m_{1}=\left(b_{1}+a_{2}\left(m_{2}-b_{2}\right) / m_{2}\right)^{2} /\left(b_{1}+a_{2}\left(m_{2}-b_{2}\right)^{2} / m_{2}^{2}\right)-o\left(b_{1}\right)$, then (A.1) can be satisfied and $Q>0$ if $b_{1} \rightarrow 0, a_{2}, b_{2}, m_{2}$ are properly chosen and either $a_{1} \rightarrow 1 / 2$ or $a_{1} \rightarrow 1$.

(ii) If we take $m_{1}=b_{1}+1$, then (A.1) can be satisfied and $Q<0$ if $b_{1} \rightarrow \infty$, $a_{2}>1, b_{2}, m_{2}$ are properly chosen and either $a_{1} \rightarrow 0$ or $a_{1} \rightarrow 1 / 2$ or $a_{1} \rightarrow 1$.

Proof. (i) As $b_{1} \rightarrow 0, m_{1} \rightarrow a_{2}$, and $A \rightarrow m_{2} /\left(m_{2}-b_{2}\right)$, it is clear that $Q>0$ provided that $b_{1} \rightarrow 0$ and either $a_{1} \rightarrow 1 / 2$ or $a_{1} \rightarrow 1$ by Proposition A.1. On the other hand, it is clear that there are $a_{2}, b_{2}, m_{2}$ such that (i) holds.

Now, we verify (ii). If $a_{1} \rightarrow 1, Q \rightarrow m_{1} m_{2}^{2}(A-1)-a_{2} b_{2}\left(m_{2}-b_{2}\right) A$. Choosing $m_{1}=$ $b_{1}+1$ and letting $b_{1} \rightarrow \infty$, we note that $A \rightarrow 1$ and $m_{1}(A-1) \rightarrow 1-a_{2}\left(m_{2}-b_{2}\right) / m_{2}$. Therefore,

$$
Q<0 \Longleftrightarrow 1-a_{2}\left(m_{2}-b_{2}\right) / m_{2}<a_{2} b_{2}\left(1-b_{2} / m_{2}\right) / m_{2}
$$

By the above choice, (A.1) becomes equivalent to

$$
\begin{aligned}
a_{2}\left(m_{2}-b_{2}\right) / m_{2} & <1, \\
1+a_{2}\left(m_{2}-b_{2}\right)^{2} / m_{2}^{2} & <2 a_{2}\left(m_{2}-b_{2}\right) / m_{2}, \\
\sqrt{a_{2}+m_{2}} & <1+\sqrt{b_{2}} .
\end{aligned}
$$

Claim. There exist $a_{2}, b_{2}, m_{2}$, and $b_{2}<m_{2}$ such that (A.2) and (A.3) are true. In fact, let $b_{2}=\alpha m_{2}$, where $\alpha \in(0,1)$ will be determined later. If $a_{2}>1$, solving (A.3), we have

$$
\left(a_{2}-1\right) / a_{2}<\alpha<\sqrt{\left(a_{2}-1\right) / a_{2}} \quad \text { and } \quad \alpha>\left(\sqrt{1+a_{2} / m_{2}}-\sqrt{1 / m_{2}}\right)^{2} .
$$

Copyright $@$ by SIAM. Unauthorized reproduction of this article is prohibited. 
It is evident that if

$$
\sqrt{\left(a_{2}-1\right) / a_{2}}>\left(\sqrt{1+a_{2} / m_{2}}-\sqrt{1 / m_{2}}\right)^{2}
$$

there is $\alpha$ such that (A.3) holds. We verify that there exist $a_{2}$ and $m_{2}$ such that (A.4) is valid. Let $a_{2}=\beta m_{2}$, where $\beta \in(0, \infty)$ will be determined later, and denote

$$
f(\beta)=\sqrt[4]{1-1 / a_{2}}+\sqrt{\beta / a_{2}}-\sqrt{1+\beta} .
$$

So, for some $\beta>0, f(\beta)>0 \Longleftrightarrow$ (A.4) holds for some $a_{2}$ and $m_{2}$. Simple analysis shows that $f(\beta)$ attains its maximal value at $\beta=1 /\left(a_{2}-1\right)$ and $f\left(1 /\left(a_{2}-1\right)\right)>0$. Take $\beta=1 /\left(a_{2}-1\right)$. According to our notation, (A.2) becomes equivalent to $a_{2}\left(1-\alpha^{2}\right)-1>0$. If $\alpha=\sqrt{\left(a_{2}-1\right) / a_{2}}, a_{2}\left(1-\alpha^{2}\right)-1=0$. Hence, we can find $\alpha$ which is close to but less than $\sqrt{\left(a_{2}-1\right) / a_{2}}$ such that (A.2) is valid. Consequently, our claim holds.

For $a_{1} \rightarrow 0$ or $a_{1} \rightarrow 1 / 2$, as above, similar analysis can be done. Therefore, from these arguments, it can follow that (ii) is also true under our requirements.

Acknowledgment. The authors wish to thank the referees for their constructive comments, which led to a significant improvement of the original manuscript.

\section{REFERENCES}

[1] P. Abrams and L. Ginzburg, The nature of predation: Prey dependent, ratio dependent or neither?, Trends Ecol. Evol., 15 (2000), pp. 337-341.

[2] D. Alonso, F. Bartumeus, and J. Catalan, Mutual interference between predators can give rise to Turing spatial patterns, Ecology, 83 (2002), pp. 28-34.

[3] K. J. Brown And F. A. Davidson, Global bifurcation in the Brusselator system, Nonlinear Anal., 24 (1995), pp. 1713-1725.

[4] T. K. Callahan and E. KNOBloch, Pattern formation in three-dimensional reaction-diffusion systems, Phys. D, 132 (1999), pp. 339-362.

[5] C. H. Chiu ANd S. B. Hsu, Extinction of top predator in a three-level food-chain model, J. Math. Biol., 37 (1998), pp. 372-380.

[6] E. Conway, D. Hoff, And J. Smoller, Large time behavior of solutions of systems of nonlinear reaction-diffusion equations, SIAM J. Appl. Math., 35 (1978), pp. 1-16.

[7] F. A. Davidson And B. P. Rynne, A priori bounds and global existence of solutions of the steady-state Sel'kov model, Proc. Roy. Soc. Edinburgh Sect. A, 130 (2000), pp. 507-516.

[8] Y. H. DU AND Y. Lou, Qualitative behavior of positive solutions of a predator-prey model: Effects of saturation, Proc. Roy. Soc. Edinburgh Sect. A, 131 (2001), pp. 321-349.

[9] H. I. Freedman And P. Waltman, Mathematical analysis of some three-species food-chain models, Math. Biosci., 33 (1977), pp. 257-277.

[10] A. Gierer, Generation of biological patterns and form: Some physical, mathematical and logical aspects, Prog. Biophys. Biol., 37 (1981), pp. 1-47.

[11] A. Hastings, T. Powell, and S. B. Hsu, Chaos in a three-species food chain, Ecology, 72 (1991), pp. 896-903.

[12] D. Henry, Geometric Theory of Semilinear Parabolic Equations, Lecture Notes in Math. 840, Springer-Verlag, Berlin, New York, 1993.

[13] S. B. Hsu, T. W. Hwang, and Y. Kuang, A ratio-dependent food chain model and its applications to biological control, Math. Biosci., 181 (2003), pp. 55-83.

[14] S. B. Hsu, H. L. Smith, And P. Waltman, Competitive exclusion and coexistence for competitive systems on ordered Banach spaces, Trans. Amer. Math. Soc., 348 (1996), pp. 4083-4094.

[15] Y. KAN-ON, Existence and instability of Neumann layer solutions for a 3-component LotkaVolterra model with diffusion, J. Math. Anal. Appl., 243 (2000), pp. 357-372.

[16] Y. KAN-ON AND M. MimURA, Singular perturbation approach to a 3-component reactiondiffusion system arising in population dynamics, SIAM J. Math. Anal., 29 (1998), pp. 1519-1536.

Copyright (c) by SIAM. Unauthorized reproduction of this article is prohibited. 
[17] A. Klebanoff and A. Hastings, Chaos in one-predator, two-prey models: General results from bifurcation theory, Math. Biosci., 122 (1994), pp. 221-233.

[18] J. A. Leach AND J. C. WeI, Pattern formation in a simple chemical system with general orders of autocatalysis and decay. I. Stability analysis, Phys. D, 180 (2003), pp. 185-209.

[19] C. S. Lin, W. M. NI, AND I. TAKAGI, Large amplitude stationary solutions to a chemotaxis system, J. Differential Equations, 72 (1988), pp. 1-27.

[20] Y. Lou, S. Martinez, and W. M. Ni, On $3 \times 3$ Lotka-Volterra competition systems with cross-diffusion, Discrete Contin. Dyn. Syst., 6 (2000), pp. 175-190.

[21] Y. LOU AND W. M. NI, Diffusion vs cross-diffusion: An elliptic approach, J. Differential Equations, 154 (1999), pp. 157-190.

[22] M. Mimura And Y. Nishiura, Pattern formation in coupled reaction-diffusion systems, Japan J. Indust. Appl. Math., 12 (1995), pp. 385-424.

[23] W. M. Ni AND I. TAKAGI, On the Neumann problem for some semilinear elliptic equations and systems of activator-inhibitor type, Trans. Amer. Math. Soc., 297 (1986), pp. 351-368.

[24] W. M. Ni AND J. C. WeI, On positive solutions concentrating on spheres for the GiererMeinhardt system, J. Differential Equations, 221 (2006), pp. 158-189.

[25] L. Nirenberg, Topics in Nonlinear Functional Analysis, American Mathematical Society, Providence, RI, 2001.

[26] K. Page, P. K. Maini, And N. A. M. Monk, Pattern formation in spatially heterogeneous Turing reaction-diffusion models, Phys. D, 181 (2003), pp. 80-101.

[27] P. Y. H. PANG AND M. X. WANG, Non-constant positive steady states of a predator-prey system with non-monotonic functional response and diffusion, Proc. London Math. Soc. (3), 88 (2004), pp. 135-157.

[28] P. Y. H. PANG AND M. X. WANG, Qualitative analysis of a ratio-dependent predator-prey system with diffusion, Proc. Roy. Soc. Edinburgh Sect. A, 133 (2003), pp. 919-942.

[29] R. Peng and M. X. Wang, Positive steady-state solutions of the Noyes-Field model for Belousov-Zhabotinskii reaction, Nonlinear Anal., 56 (2004), pp. 451-464.

[30] R. Peng and M. X. Wang, Pattern formation in the Brusselator system, J. Math. Anal. Appl., 309 (2005), pp. 151-166.

[31] R. Peng and M. X. Wang, Positive steady-states of the Holling-Tanner prey-predator model with diffusion, Proc. Roy. Soc. Edinburgh Sect. A, 135 (2005), pp. 149-164.

[32] R. Peng And M. X. Wang, On pattern formation in the Gray-Scott model, Sci. China Ser. A, 50 (2007), pp. 377-386.

[33] M. L. Rosenzweig, Paradox of enrichment: Destabilization of exploitation systems in ecological time, Science, 171 (1969), pp. 385-387.

[34] A. Turing, The chemical basis of morphogenesis, Philos. Trans. R. Soc. Lond. Ser. B., 237 (1952), pp. 37-72.

[35] M. X. WANG, Non-constant positive steady states of the Sel'kov model, J. Differential Equations, 190 (2003), pp. 600-620.

[36] M. X. WANG, Stationary patterns for a prey-predator model with prey-dependent and ratiodependent functional responses and diffusion, Phys. D, 196 (2004), pp. 172-192.

[37] J. C. WEI AND M. WinTER, Existence and stability of multiple-spot solutions for the Gray-Scott model in $R^{2}$, Phys. D, 176 (2003), pp. 147-180.

Copyright (c) by SIAM. Unauthorized reproduction of this article is prohibited. 


\title{
ERRATUM: STATIONARY PATTERN OF A RATIO-DEPENDENT FOOD CHAIN MODEL WITH DIFFUSION*
}

\author{
RUI PENG ${ }^{\dagger}$, JUNPING SHI ${ }^{\ddagger}$, AND MINGXIN WANG§
}

Abstract. The main purpose of this erratum is to correct an error in the proof of Theorem 4.4 in [R. Peng, J. Shi, and M. Wang, SIAM J. Appl. Math., 67 (2007), pp. 1479-1503].

Key words. food chain model, diffusion, stationary pattern

AMS subject classifications. 35J55, 35K57, 92C15, 92C40

DOI. $10.1137 / 090752845$

In the proof of Theorem 4.4 of [1, pages 1492-1493], we used the method of the implicit function theorem. On page 1493, in the course of constructing the operator $F$, we made an error, because for the operator $F$ defined there, we cannot guarantee that the range of the second component $f_{2}$ is in $L_{0}^{2}(\Omega)$. We now need to make a minor change to the definition of the operator $F$ as follows.

To this end, we first introduce the projection operator from $L^{2}(\Omega)$ to $L_{0}^{2}(\Omega)$. That is, for any $g \in L^{2}(\Omega)$, we set

$$
\mathbf{P}(g)=g-\frac{1}{|\Omega|} \int_{\Omega} g \mathrm{~d} x .
$$

Then the corrected operator $F$ should be given in the following form:

$$
F\left(d_{1}, d_{3}, \rho, u_{1}, w_{2}, \xi, u_{3}\right)=\left(f_{1}, f_{2}, f_{3}, f_{4}\right)\left(d_{1}, d_{3}, \rho, u_{1}, w_{2}, \xi, u_{3}\right),
$$

with the same definitions for $f_{1}, f_{3}$, and $f_{4}$ as on page 1493 , but the definition of $f_{2}$ is changed to

$f_{2}\left(d_{1}, d_{3}, \rho, u_{1}, w_{2}, \xi, u_{3}\right)=\Delta w_{2}+\rho \mathbf{P}\left\{\frac{m_{1} u_{1}\left(w_{2}+\xi\right)}{u_{1}+w_{2}+\xi}-b_{1}\left(w_{2}+\xi\right)-\frac{a_{2}\left(w_{2}+\xi\right) u_{3}}{w_{2}+\xi+u_{3}}\right\}$.

Then

$$
\begin{aligned}
F: & \mathbf{R}^{+} \times \mathbf{R}^{+} \times \mathbf{R}^{+} \times W_{\nu}^{2,2}(\Omega) \times\left(L_{0}^{2}(\Omega) \cap W_{\nu}^{2,2}(\Omega)\right) \times \mathbf{R}^{+} \times W_{\nu}^{2,2}(\Omega) \\
& \rightarrow L^{2}(\Omega) \times L_{0}^{2}(\Omega) \times \mathbf{R} \times L^{2}(\Omega)
\end{aligned}
$$

is well defined and the remaining part of the proof for assertion (i) of Theorem 4.4 can proceed as in the paper. For the proof of assertion (ii) of Theorem 4.4, a similar but obvious modification is also needed for the operator $F$ defined on page 1494 .

\section{REFERENCE}

[1] R. Peng, J. Shi, And M. Wang, Stationary pattern of a ratio-dependent food chain model with diffusion, SIAM J. Appl. Math., 67 (2007), pp. 1479-1503.

${ }^{*}$ Received by the editors March 17, 2009; accepted for publication (in revised form) April 3, 2009; published electronically June 10, 2009.

http://www.siam.org/journals/siap/70-1/75284.html

$\dagger$ Institute of Nonlinear Complex Systems, College of Science, China Three Gorges University, Yichang, 443002, Hubei, P. R. China (pengrui_seu@163.com).

${ }^{\ddagger}$ Department of Mathematics, College of William and Mary, Williamsburg, VA 23187-8795, and Department of Mathematics, Harbin Normal University, Harbin, 150080, Helongjiang, P. R. China (shij@math.wm.edu).

$\S$ Department of Mathematics, Southeast University, Nanjing, 210018, Jiangsu, P. R. China (mxwang@seu.edu.cn). 